\title{
Red Blood Cells Under Flow Show Maximal ATP Release for Specific Hematocrit
}

\author{
Zhe Gou ${ }^{1}$, Hengdi Zhang ${ }^{1,2}$, Mehdi Abbasi ${ }^{1}$, and Chaouqi Misbah ${ }^{1, *}$ \\ ${ }^{1}$ Université Grenoble Alpes, CNRS, LIPhy, Grenoble, France \\ ${ }^{2}$ Shenzhen Sibionics Co. Ltd., Shenzhen, China \\ *Correspondence: chaouqi.misbah@univ-grenoble-alpes.fr
}

\begin{abstract}
ATP release by red blood cells (RBCs) under shear stress (SS) plays a pivotal role in endothelial biochemical signaling cascades. The aim of this study is to investigate through numerical simulation how does RBCs spatio-temporal organization depend on flow and geometrical conditions to generate ATP patterns. Numerical simulations were conducted in a straight channel by considering both plasma and explicit presence of RBCs, their shape deformation and cell-cell interaction, as well as ATP release by RBCs. Two ATP release pathways through cell membrane are taken into account, pannexin 1 channel (Px1), sensitive to SS, and cystic fibrosis transmembrane conductance regulator (CFTR) which responds to cell deformation. Several flow and hematocrit conditions are explored. The problem is solved by lattice Boltzmann method. Application of SS to the RBC suspension triggers a nontrivial spatial RBC organization and ATP patterns. ATP localizes preferentially in the vicinity of cell-free layer close to channel wall. Conditions for maximal ATP release per cell are identified, which depend on vessel size and hematocrit $H t$. Increasing further $H t$ beyond optimum enhances the total ATP release but should degrade oxygen transport capacity, a compromise between an efficient ATP release and minimal blood dissipation. Moreover, ATP is boosted in capillaries suggesting a vasomotor activity coordination throughout the resistance network.
\end{abstract}

SIGNIFICANCE Red Blood Cells (RBCs) are not only oxygen cargoes but transport ATP as well. ATP is released under sufficient RBCs mechanical stress and deformation. Released ATP react with vascular walls to trigger cascades of chemical reactions, such as calcium waves, to regulate blood perfusion. Numerical simulation of RBCs under flow yields a general picture of RBCs organization in a vessel and the corresponding ATP pattern. The study reveals a given hematocrit for maximal ATP release in arterioles and that ATP is boosted in capillaries. This points to a subtle role of RBCs spatio-temporal organization in blood flow regulation, shedding a new light on implication of RBCs in biochemical signaling.

\section{INTRODUCTION}

ATP is a key regulatory component for many cell functions (1), not only for intracellular but also for extracellular functions. A prominent example for the latter involves red blood cells (RBCs) which may release ATP under flow to regulate vasotone response $(2,3)$. When a sufficient amount of ATP is released from RBCs a reaction with purinergic receptors (a class of membrane receptors that mediates vascular reactivity, etc.) $(4,5)$ on the surface of endothelial cells (ECs) leads to an intracellular calcium/eNOS (endothelial nitric oxide synthase) signal. This signal can be transmitted to neighboring ECs (in the form of waves) ultimately giving rise to vasodilation. Several diseases have been linked to insufficient ATP release from RBCs, such as cystic fibrosis (6) and type 2 diabetes $(7,8)$. Elucidating the mechanism of ATP release by RBCs is a basic step for understanding the interplay between blood flow and biochemical signaling, and ultimately to design appropriate therapies.

Several in vitro experimental studies suggest that the release of ATP is triggered by shear stress (9-11). Two pathways have been identified. The first one involves a mechanosensitive channel, the pannexin 1 hemichannel (Px1), which is considered as a main candidate for ATP release, when exposed to hypotonic stress (12). The second pathway is related to ample RBCs deformation under flow resulting in the activation of the cystic fibrosis transmembrane conductance regulator (CFTR) (13), which eventually regulates the release of ATP (6). CFTR is believed to indirectly act on ATP by upregulating Px1 channel (14). Following these two scenarios, opening of Px1 channel due to a critical shear stress, and its upregulation (due to sufficient RBCs 
deformation), a theoretical model, combined with numerical simulations, successfully (15) accounted for in vitro experiments (14) conducted on a single RBC under shear flow.

In real situations hematocrit $(H t)$ is finite and RBCs interact with each other in a nontrivial manner. This results in a complex spatio-temporal organization. A particularly documented phenomenon is the Fahraeus effect (16-18) which leads to a cell-free layer (CFL) in the vicinity of blood vessel walls, especially in microcirculations. This has dramatic consequences on blood transport, since blood viscosity decreases, due to CFL, by many folds with decreasing vascular diameter. In addition, in microcirculation RBCs tend to form clusters, which in turn lead to an increase of viscosity at low shear rates (19). The RBCs patterns and their implication in blood flow properties is expected to critically impact the ATP release process. The main goal of this paper is to analyze the link between RBCs organization and its relation to ATP release.

We investigate here, with the help of a systematic numerical simulation, the ATP release pattern by RBCs in a pressure driven flow. We explore the role of flow strength, as well as $H t$ and vessel diameter. We pinpoint several features, such as the emergence of a maximal ATP release for a given hematocrit and provide a general physical interpretation.

\section{METHODS}

\section{Mathematical model}

The details of the mathematical model can be found in (15, 20-22). Here we provide a brief account for completeness. As shown in Figure 1, RBCs are immersed in a straight channel filled with plasma. We use a 2D model for computational efficiency, without compromising the main result (as proven in $(15,20)$ ). Indeed in that paper it has been shown that the 2D model captures the essential features observed in in vitro experiments (14). To complete the presentation, we extend our results to 3D. For the mechanical part in 3D we adopt the model proposed by Krüger et al. (21, 22). From the knowledge of cell configurations and stress field, we can calculate the ATP release rate from RBCs. In this work, we leave the ATP evolution (i.e. how ATP is distributed in the channel) for a future work. Since 2D and 3D simulations provide the same main qualitative features, most of results focus on 2D, and 3D simulations are shown as complement. The channel length and width are $L$ and $W$, respectively (in 3D, the cross section of the channel is a square with side width $W$ ). The cell membranes are denoted as $\Gamma$ and the fluid domain is divided into $\Omega_{i n}$ and $\Omega_{e x}$, representing the domain inside and outside the cells, respectively. The coordinates of RBCs and fluid are denoted by $\mathbf{X}(s)$ and $\mathbf{x}(x, y)(\mathbf{x}(x, y, z)$ in 3D) respectively, where $s$ is the curvilinear coordinate (in 2D) or surface coordinate (in $3 \mathrm{D}$ ) along the cell membrane.

The fluid dynamics inside and outside RBCs is governed by the Navier-Stokes equations:

$$
\begin{aligned}
& \rho\left(\frac{\partial \mathbf{u}}{\partial t}+\mathbf{u} \cdot \nabla \mathbf{u}\right)=-\nabla p+\nabla \cdot\left[\mu\left(\nabla \mathbf{u}+\nabla \mathbf{u}^{T}\right)\right]+\mathbf{f}, \\
& \nabla \cdot \mathbf{u}=0,
\end{aligned}
$$

where $\rho, \mathbf{u}$ and $p$ are the fluid density, velocity and pressure, respectively. The viscosity $\mu$ can take different values for interior and exterior fluids as

$$
\mu(\mathbf{x})= \begin{cases}\mu_{i n}, & \mathbf{x} \in \Omega_{i n}, \\ \mu_{e x}, & \mathbf{x} \in \Omega_{e x}\end{cases}
$$

and the viscosity ratio is defined as $\lambda=\mu_{i n} / \mu_{e x}$. The source term $\mathbf{f}$ in equation 1 represents the effect of cell membrane on the fluid (see below). A pressure gradient is applied between inlet and outlet, resulting in an undisturbed (i.e. in the absence of $\mathrm{RBCs}$ ) Poiseuille flow $\mathbf{u}=(u, v)$ (in 2D):

$$
\begin{aligned}
& u=u_{\max }\left(1-\frac{4 y^{2}}{W^{2}}\right), \\
& v=0,
\end{aligned}
$$

where $u_{\max }$ is the maximum velocity at the center line located at $y=0$.

The cell membrane mechanical properties are given by the Helfrich bending energy (23) as

$$
E_{H}(\mathbf{X}(s))=\frac{\kappa}{2} \int_{\Gamma} c^{2}(s) d s+\int_{\Gamma} \zeta(s) d s,
$$

where $\kappa$ is the membrane bending modulus, $c$ is the local RBC curvature, and $\zeta$ is a Lagrange multiplier to enforce local membrane inextensibility. The membrane force in 2D can be obtained by functional derivative of the energy (24) as

$$
\mathbf{F}(s)=\frac{\delta E_{H}}{\delta \mathbf{X}(s)}=\kappa\left(\frac{\partial^{2} c}{\partial s^{2}}+\frac{c^{3}}{2}\right) \mathbf{n}-c \zeta \mathbf{n}+\frac{\partial \zeta}{\partial s} \mathbf{t},
$$




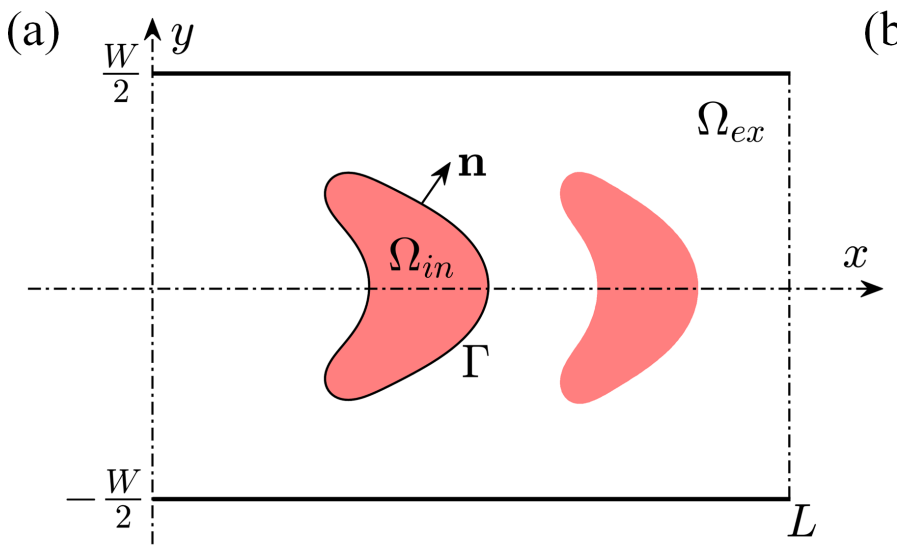

(b)

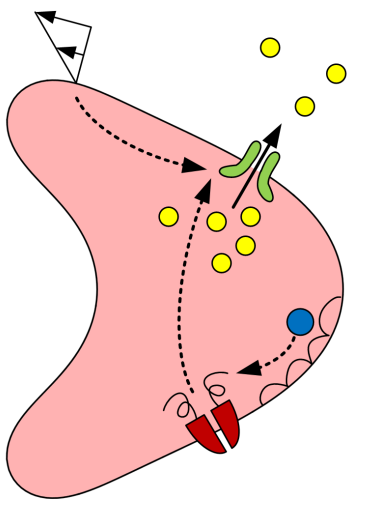

\section{○ ATP \\ $\int \mathrm{Px} 1$}

Freed Actin

96 CFTR

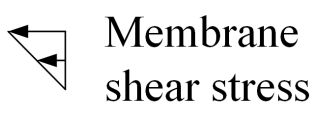

(c)

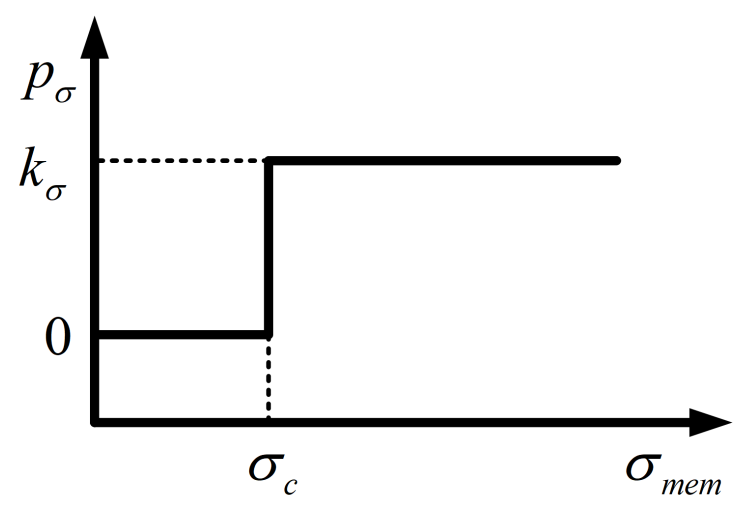

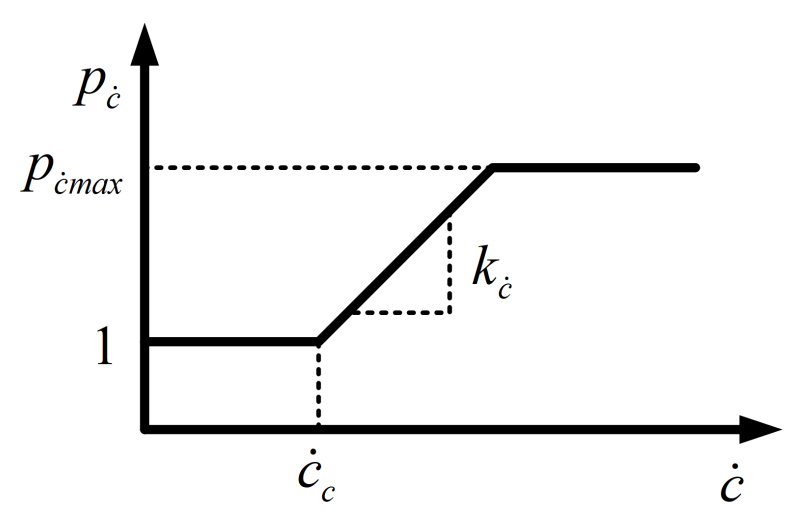

Figure 1: (a) Schematic of the studied configuration: RBCs immersed in a straight channel filled with plasma. $L$ and $W$ are the channel length and width, respectively. $\Omega_{i n}$ and $\Omega_{e x}$ refer to the interior and exterior fluid domains. $\mathbf{n}$ is the outward normal vector, and $\Gamma$ is the cell membrane. The solid straight lines refer to the channel walls. For simplicity, $\Omega_{i n}, \Gamma$, and $\mathbf{n}$ are only marked for one cell. (b) Schematic of the ATP release pathway in RBC: Px1 is the channel for intracellular ATP release, which can be activated by membrane shear stress; membrane deformation results in free actin, which can activate CFTR and consequently upregulate Px1. (c) Schematics of the shear stress dependent function (left panel) and the deformation dependent function (right panel).

where $\mathbf{n}$ and $\mathbf{t}$ are the outward normal and tangential unit vector, respectively. In $3 \mathrm{D}$, the membrane has, in addition, a shear elastic energy written as $\kappa_{s}\left(I_{1}^{2}+2 I_{1}-I_{2}\right) / 12+\kappa_{\alpha} I_{2}^{2} / 12$, where $\kappa_{s}$ is the shear elastic modulus, $\kappa_{\alpha}$ is the area dilation modulus. $I_{1}$ and $I_{2}$ are the in-plane strain invariants (see Ref. (21)). The calculation of membrane force in 3D follows the description in Ref. (21).

The immersed boundary method (25) is adopted to deal with the interaction between cells and fluid. The force exerted by cell membrane on the fluid is calculated through the following spread equation:

$$
\mathbf{f}=\int_{\Gamma} \mathbf{F}(s) \boldsymbol{\delta}(\mathbf{x}-\mathbf{X}(s)) d s
$$

Here $\delta(\mathbf{x})$ is a regularized Dirac delta function. A no-slip condition is adopted on the cell membrane and its velocity $\mathbf{U}$ is interpolated from the fluid velocity:

$$
\frac{d \mathbf{X}}{d t}=\mathbf{U}(s)=\int_{\Omega} \mathbf{u}(\mathbf{x}) \boldsymbol{\delta}(\mathbf{x}-\mathbf{X}(s)) d \mathbf{x}
$$

The evolution of ATP is described by the advection-diffusion equation as

$$
\frac{\partial a}{\partial t}+\mathbf{u} \cdot \nabla a=\nabla \cdot(D \nabla a)
$$

where $a$ is the ATP concentration and $D$ is the ATP diffusion coefficient in plasma. It should be noted that the ATP is released by the cell membrane towards the plasma. Thus, only ATP concentration in $\Omega_{e x}$ is solved explicitly, and the inside of RBCs is 
considered as a homogeneous reservoir of ATP. This is justified by the fact that the ATP concentration inside RBCs is typically in the range of $\mathrm{mM}$, whereas that in plasma is in the range of $\mu \mathrm{M}$. Under shear stress RBCs can release ATP. The ATP flux across the membrane is written as

$$
D \frac{\partial a}{\partial \mathbf{n}}=-\psi
$$

$\psi$ is a source term, expressing the rate of ATP release by the cell. This rate depends, as described below, on shear stress, and cell deformation, the two main pathways of ATP release. It should be noted that equations 8 and 9 are solved for 2D simulations only. In 3D, the ATP flux $\psi$ is calculated, without solving the advection-diffusion equation. This is sufficient in order to show that the main result, namely the existence of a maximal ATP release for a given $H t$ is also exhibited in 3D. The solution of the advection-diffusion equation allows to describe how the released ATP is distributed in space and time. In 3D, this task will be postponed to the future.

We consider a one-way coupling between blood flow and ATP release. In other words, the blood flow can induce ATP release from RBCs, while an increase of plasma ATP concentration is supposed not to affect fluid dynamics. We are not aware of a direct effect of ATP on viscosity of blood suspension. ATP may affect, for example, RBCs deformation due to its active effect on cytoskeleton (26). Plasma proteins, like fibrinogen, can lead to the formation of robust clusters of RBCs at low shear rates $\left(<1 \mathrm{~s}^{-1}\right)$ or stasis. In some pathological conditions, the increase of fibrinogen concentration may induce RBC aggregation even at large shear rates. In the present work, we consider a wall shear rate in the $50 \sim 1000 \mathrm{~s}^{-1}$ range in healthy conditions. We hope to report on RBCs aggregation on ATP release in the future.

\section{ATP release model}

As described in the Introduction, two mechanisms are adopted for the release of ATP: (i) a sufficient membrane shear stress triggers ATP release through Px1 channel, and (ii) the increase of membrane deformation further amplifies the release by modulating CFTR to upregulate Px1 channel (see Figure 1b). The phenomenological model proposed by Zhang et al (15) is adopted in this work to calculate ATP released by RBCs. For each membrane element, the local shear stress is defined as $\sigma_{m e m}(s)=\mu_{e x}\left|\partial u_{\mathbf{t}}(\mathbf{X}(s)) / \partial \mathbf{n}\right|$, where $u_{\mathbf{t}}$ is the velocity along the tangential direction of the membrane. The ATP release rate due to Px1 channels is given as (see Figure 1c, left panel)

$$
p_{\sigma}=k_{\sigma} \cdot H\left(\sigma_{m e m}-\sigma_{c}\right)
$$

where $H$ is the Heaviside function, $k_{\sigma}$ is the ATP release coefficient, and $\sigma_{c}$ is the critical shear stress. $p_{\sigma}$ has a dimension of a concentration flux, namely $(\mathrm{nM}) \cdot \mathrm{m} / \mathrm{s}$ (nM stands for nanomolar). This equation simply means that ATP is released only if the local shear stress exceeds a certain value $\sigma_{c}$. In the in vitro experiment of Ref. (11) it has been reported that $\sigma_{c}$ is estimated to lie in the range $0.02-0.1 \mathrm{~Pa}$, the lowest range in microcirculation. We have set it to $0.05 \mathrm{~Pa}$ (see Table 1 ). This would mean that Px1 would always be activated as long as membrane shear stress is higher than this value, which is relatively small. However, not all channels on a given cell will be activated since the hydrodynamics stress along the membrane is heterogeneous so that some parts of the RBC membrane will fall below the critical value. In addition cells in the middle of the channel experience a quite low shear stress so that no Px1 (or at best a low proportion of them) will be activated. It will be seen that despite the low value of $\sigma_{c}$ the pattern of ATP release is quite diverse depending on site of microcirculation.

Some remarks are in order about how the ATP channel sense mechanical stress. Pannexins are transmembrane glycoproteins. The precise question on how Px1 sense mechanical stress is not completely clear. For example, if sensing occurs via the molecular part which is pending in the surrounding fluid, then it is likely that the applied hydrodynamics stress, as adopted here, is decisive. If on the contrary, the sensing takes place in the part of the molecule which is embedded inside the membrane, then membrane tension should play a major role. This last model was adopted in Ref. (27) for the problem of gating of mechanosensitive channels under flow. Different channel sensing scenario are discussed in Ref. (28).

The membrane deformation is characterized by its local curvature change rate. The membrane deformation rate is defined as the Lagrangian derivative of curvature: $\dot{c}(s, t)=|d c(s, t) / d t|$. The contribution of CFTR is modeled as an amplifier to Px1 (see Figure 1c, right panel):

$$
p_{\dot{c}}=\min \left(1+k_{\dot{c}} \cdot\left(\dot{c}-\dot{c}_{c}\right) \cdot H\left(\dot{c}-\dot{c}_{c}\right), p_{\dot{c}_{\max }}\right),
$$

where $k_{\dot{c}}$ is a phenomenological coefficient, $\dot{c}$ and $\dot{c}_{c}$ are the local and critical curvature change rate, and $p_{\dot{c}_{\max }}$ is an upper limit. As shown in Figure 1b, membrane deformation results in free actin, which eventually activates CFTR. How RBC deformation leads to free actin is still an unsolved question. There are two main hypotheses. (i) The deformation of cell membrane results in defects of spectrin network. These defects form buckled pyramids of spectrin molecules, detached from actin node (29). (ii) The deformation of cell membrane leads to extreme stretching and unfolding of spectrin filaments, which makes the actin segments more accessible to the CFTR molecules (30-32). In the present study, we adopt the first hypothesis, assuming that 
the defects are positively related to membrane deformation. The existence of an upper limit, expressed by $p_{\dot{c}_{\max }}$, is based on the notion that there should exist a maximal amount of free actin and a limited level of activatable CFTR. According to equation $11, p_{\dot{c}}$ is dimensionless and its value varies between 1 and $p_{\dot{c}_{\max }}$. ATP will still be released even if $p_{\dot{c}}=1$, meaning that only shear stress would be responsible for ATP release. The phenomenological parameters entering the model have been extracted by comparison with experiments (15) (see Table 1).

The overall rate of ATP production, entering equation 12, combines the two above processes resulting into

$$
\psi=p_{\sigma} p_{\dot{c}} .
$$

As shown in equation 12, $\psi$ is the product of $p_{\sigma}$ and $p_{\dot{c}}$, and has the unit of concentration flux as $(\mathrm{nM}) \cdot \mathrm{m} / \mathrm{s}$. In the absence Px1 activation (that is $p_{\sigma}=0$ ), there is no ATP release. The membrane deformation can modulate CFTR to upregulate (or amplify) the Px1 channel, which leads to the increase of ATP release rate $\left(p_{\dot{c}}>1\right)$. The ATP chemical potential is related to concentration difference across the cell membrane. The resulting ATP flux across the membrane is $\psi=g\left(a_{i n}-a_{e x}\right) p_{\sigma} p_{\dot{c}}$, where $g\left(a_{i n}-a_{e x}\right)$ is a function related to ATP concentration inside $\left(a_{i n}\right)$ and outside $\left(a_{e x}\right)$ the cell. As stated in previous section, the typical range of intracellular ATP concentration is $\sim 1 \mathrm{mM}$. In the present study, the highest plasma ATP concentration observed in the smallest vessel is at the level of $\sim 1 \mu \mathrm{M}$. This value is three orders of magnitude smaller than the intracellular ATP concentration. Hence it is reasonable to consider $g\left(a_{i n}-a_{e x}\right)$ as a constant, neglecting the effect of chemical potential. For a single RBC, the total ATP release rate is the integral of equation 12 over the cell membrane:

$$
\psi_{\mathrm{RBC}}=\int_{0}^{P} \psi(\sigma, \dot{c}) d s,
$$

where $P$ is the perimeter (in 2D) or the surface area (in 3D) of the cell. The present model has been inspired by in vitro experiments (14) which pointed to the fact that the stress activates Px1 channels, whereas deformation upregulates (amplifies) ATP release. The model has been successfully confronted with experiments (15).

\section{Simulation set-up and analysis}

An immersed-boundary-lattice-Boltzmann method is implemented to solve the fluid and cell dynamics (20-22). Using a typical RBC radius $R_{0} \sim 3 \mu \mathrm{m}$, plasma viscosity (close to that of water) $\mu_{e x} \sim 10^{-3} \mathrm{~Pa} \cdot \mathrm{s}$ (and density $\rho \sim 10^{3} \mathrm{~kg} / \mathrm{m}^{3}$ ), and shear rate in microcirculation, $\dot{\gamma} \sim 10-1000 \mathrm{~s}^{-1}$, we find that the typical Reynolds number $R e=\rho R_{0}^{2} \dot{\gamma} / \mu_{e x}$ is about 0.0001 to 0.01 . Within this range, the flow is in the viscous regime and inertia effects are negligible. However, using these small values of $R e$ would dramatically slow down the numerical computation. Several benchmarks $(15,20,33-35)$ have been made in the literature in order to define a maximal value of $R e$ below which the lattice-Boltzmann method reproduces with a good precision the Stokes regime. There is a consensus that a value $R e=0.1$ is a good compromise between numerical efficiency and good precision for the Stokes regime. For this reason, the Reynolds number is set in our simulation to 0.1. For ATP, we adopted a similar lattice-Boltzmann method $(15,36)$ to solve the advection-diffusion equation. The ATP release at the cell membrane is treated using a modified bounce-back boundary condition for moving boundaries.

For a cell with perimeter $P$ and area $A$ in 2D, its characteristic radius is defined as $R_{0}=\sqrt{A / \pi}$, which equals the radius of the circle with the same area. In 3D, the cell surface area and volume are $P$ and $V$, respectively. The characteristic radius is $R_{0}=\sqrt[3]{3 V / 4 \pi}$. The reduced area (in 2D) is defined as $\tau=4 \pi A / P^{2}$, and the reduced volume (in 3D) is $\tau^{\prime}=(4 \pi)^{1 / 2} \cdot 3 V / P^{3 / 2}$ to measure the degree of deflation. In the present work, we set $R_{0}=3 \mu \mathrm{m}$ and $\tau=\tau^{\prime}=0.65$ for all cases, which are characteristic values of human RBCs. For all simulations, the channel length is fixed at $L=30 R_{0}=90 \mu \mathrm{m}$ in $2 \mathrm{D}$ and $L=15 R_{0}=45 \mu \mathrm{m}$ in 3D, which was validated to be long enough to neglect the effect of periodic boundary condition between inlet and outlet. Different values of width $W$ are explored, and the degree of confinement is defined as $C n=2 R_{0} / W$. The exterior viscosity is set as $\mu_{e x}=0.001 \mathrm{~Pa} \cdot \mathrm{s}$ (close to that of plasma). As previously mentioned, the viscosity ratio of the interior and exterior fluids is $\lambda$. For all simulations, the viscosity ratio is fixed as $\lambda=6$, a well adopted value for human RBCs. In order to characterize the fluid flow, the capillary number $C a=\mu_{e x} \dot{\gamma}_{w} R_{0}^{3} / \kappa$ is defined, where $\dot{\gamma}_{w}=4 u_{\max } / W$ refers to the shear rate at the channel wall. The capillary number represents the ratio between fluid shear stress energy and the resistance of cell membrane to bending. We take a typical value for RBCs reported in literature (26) $\kappa=3 \times 10^{-19} \mathrm{~J}$. In 3D, we take $\kappa_{s}=4 \mu \mathrm{N} / \mathrm{m}$, close to that known for RBCs (37), and $\kappa_{\alpha} / \kappa_{s}=200$ to ensure local area conservation.

The effective viscosity of cell suspension is defined by

$$
\mu_{e f f}=\frac{\mu_{e x} Q}{Q_{s s p}},
$$

where $Q_{s s p}$ is the average flux of cell suspension, and $Q$ is the flux of the pure plasma (with viscosity $\mu_{e x}$ ) when subjected to the same pressure gradient. To evaluate the contribution of RBCs to the viscosity of the suspension, we define the intrinsic 
Gou et al.

viscosity as

$$
[\mu]=\frac{\mu_{e f f}-\mu_{e x}}{\mu_{e x} H t} .
$$

As for the calculation of ATP, the diffusion coefficient of ATP in plasma is $D=2.36 \times 10^{-10} \mathrm{~m}^{2} / \mathrm{s}$, based on the study of John et al (38). The initial ATP concentration in plasma is set at 0 . The diffusion of ATP is quantified by the Peclet number as $P e=\dot{\gamma}_{w} R_{0}^{2} / D$. For the ATP release model, we take the parameters estimated by Zhang et al (15) with an exception for $k_{\sigma}$, as shown in Table 1. In that work, $k_{\sigma}$ was estimated according to the experimental results of Forsyth et al (14) and a typical plasma ATP concentration of $1000 \mathrm{nM}$ (39). However in their experiments (14), the blood was diluted for about 40 times to obtain a RBC suspension with hematocrit $H t=1 \%$. This results in an ATP dilution as well.

Table 1: Parameters for ATP release model

\begin{tabular}{lc}
\hline Notation & Value \\
\hline$\dot{c}_{c}$ & $200 \mu \mathrm{m}^{-1} \mathrm{~s}^{-1}$ \\
$k_{\dot{c}}$ & $0.006 \mu \mathrm{m} \cdot \mathrm{s}$ \\
$p_{\dot{c}_{\max }}$ & 2.5 \\
$\sigma_{c}$ & $0.05 \mathrm{~Pa}$ \\
$k_{\sigma}$ & $175(\mathrm{nM}) \cdot \mu \mathrm{m} / \mathrm{s}$ \\
\hline
\end{tabular}

We define a reference level of the total ATP release of an RBC when all membrane elements have reached $\sigma_{1} \geq \sigma_{c}$ and $\dot{c}_{1} \leq \dot{c}_{c}$ (meaning all Px1 channels are activated but not CFTR), which is given by $\psi_{1}=\int_{0}^{P} \psi\left(\sigma_{1}, \dot{c}_{1}\right) d s=k_{\sigma} P$. With this reference value, the normalized ATP release level for a single cell is defined as

$$
\psi^{*}=\frac{\psi_{\mathrm{RBC}}}{\psi_{1}}
$$

It should be mentioned that for multi-cell simulations, we use the notation * to represent the average value along a given cell membrane, and \langle\rangle for the average value over all cells and over time. The average values of different useful quantities are listed in Table 2 for clarity. Here $i$ refers to the $i$ th cell, $N_{c}$ is the total number of cells, and $N_{y}$ is the number of cells at $y$ position. The time window $T$ is set to satisfy $\dot{\gamma}_{w} T>500$ and includes more than 100 time steps.

\begin{tabular}{|c|c|c|}
\hline Notation & Equation & Physical meaning \\
\hline$\sigma_{m e m}^{*}$ & $\frac{1}{P} \int_{0}^{P} \sigma_{m e m} d s$ & Average membrane shear stress of a given cell at a given time \\
\hline$\left\langle\sigma_{m e m}^{*}\right\rangle$ & $\frac{1}{T} \int_{0}^{T} \sum_{i}^{N_{c}} \frac{\sigma_{m e m, i}^{*}}{N_{c}} d t$ & Average membrane shear stress over all cells and over time \\
\hline$\dot{c}^{*}$ & $\frac{1}{\dot{c}_{c} P} \int_{0}^{P} \dot{c} d s$ & Average curvature change of a given cell at a given time \\
\hline$\left\langle\dot{c}^{*}\right\rangle$ & $\frac{1}{T} \int_{0}^{T} \sum_{i}^{N_{c}} \frac{\dot{c}_{i}^{*}}{N_{c}} d t$ & Average curvature change over al \\
\hline$\psi^{*}$ & $\frac{1}{k_{\sigma} P} \int_{0}^{P} \psi d s$ & Average ATP release level of a given cell at a given time \\
\hline$\left\langle\psi^{*}\right\rangle$ & $\frac{1}{T} \int_{0}^{T} \sum_{i}^{N_{c}} \frac{\psi_{i}^{*}}{N_{C}} d t$ & Average ATP release level over all cells and over time \\
\hline$\left\langle\psi^{*}\right\rangle_{y}$ & $\frac{1}{T} \int_{0}^{T} \sum_{i}^{N_{y}} \frac{\psi_{i}^{*}}{N_{y}} d t$ & Average ATP release level over cells at $y$ position and over time \\
\hline
\end{tabular}

Table 2: Average values of parameters

\section{RESULTS AND DISCUSSION}

Here, we focus on ATP released by RBCs in straight channels, leaving the computationally expensive study of complex vascular network to the future. The influence of three main parameters are explored (corresponding to a typical range in microcirculation; see Table 3): capillary number $\mathrm{Ca}$, hematocrit $\mathrm{Ht}$, and confinement $\mathrm{Cn}$. We offer a conversion between dimensionless and physiological numbers in Table 3. These values are not those provided in Ref. (40), but remain in a reasonably close enough range (for example, our arteriole diameter, $45 \mu \mathrm{m}$, lies within their range, 15-60 $\mu \mathrm{m}$ ). Note that since here we vary capillary number in a quite significant range, it is equivalent to varying diameter and/or shear rate, meaning that our data scan, for a given type of vessel, a quite significant range of values. The simulations are performed until a permanent regime is established $\left(\dot{\gamma}_{w} t>1000\right)$, at which the ATP release level per cell $\left\langle\psi^{*}\right\rangle$ varies in a small range with time. 
Table 3: Conversion between dimensionless and physiological numbers

\begin{tabular}{lccccc}
\hline & Diameter $(\mu \mathrm{m})$ & Mean velocity* $(\mathrm{mm} / \mathrm{s})$ & Wall shear stress** & $C n$ & $C a$ \\
\hline Arterioles & 45 & 7.5 & 1 & 0.133 & 90 \\
& 15 & 2.5 & 1 & 0.4 & 90 \\
Capillaries & 7.5 & 0.625 & 0.5 & 0.8 & 45 \\
Venules & 15 & 0.25 & 0.1 & 0.4 & 9 \\
& 45 & 1.875 & 0.25 & 0.133 & 22.5 \\
\hline
\end{tabular}

${ }^{*}$ Estimated with the undisturbed maximum velocity $u_{\text {mean }}=2 u_{\max } / 3$.

${ }^{* *}$ Estimated as $\tau_{w}=6 \mu_{e x} u_{m e a n} / W$.

\section{RBC dynamics and ATP release}

We first consider a channel width of $W=15 R_{0}=45 \mu \mathrm{m}$, which falls approximately in the range of arterioles and venules (40, 41). The corresponding confinement is $C n=0.133$. At initial time, 66 cells with a biconcave shape are evenly distributed in the channel, corresponding to a hematocrit of $46.0 \%$. After $0.576 \mathrm{~s}$ of flow with a capillary number of 90 , a permanent distribution of RBCs is observed (see Figure 2a). At first sight (from color code), ATP concentration seems to be lower close to the walls (at the CFL; Figure 2a). Actually, the RBCs occupy a large portion of the fluid domain in the core region, so that the ATP, which is present only in the interstitial regions between cells, is overall less abundant in the core than in the CFL if we count the area occupied by RBCs. In addition, cells close to the CFL experience a higher shear stress than in the core region, implying more ATP release close to the walls. To quantify this phenomenon, we evaluate the ATP concentration along the flow direction (averaged over the channel length), defined as

$$
\bar{a}(y)=\frac{1}{L} \int_{0}^{L} a(x, y) d x,
$$

and plotted as the red solid line in Figure 2b. We clearly see that at the two-extreme regions of that figure (corresponding to CFL) the amount of ATP is higher than the average in the core region. This can be explained by the following two reasons. (i) In a Poiseuille flow, the shear stress is higher as the cell gets closer to the boundaries. As a consequence, the ATP release rate is also higher for those cells (see Figure S1 in supplementary material). Because of the crowding of cells in the middle of the channel, the convection and diffusion of ATP towards the center are reduced. (ii) In the center of the channel, most of the volume is occupied by the cells, where the ATP concentration is 0, meaning an overall lower ATP concentration. The presence of a higher amount of ATP in the vicinity of walls (CFL) favors a fast transfer to ECs.

(a)

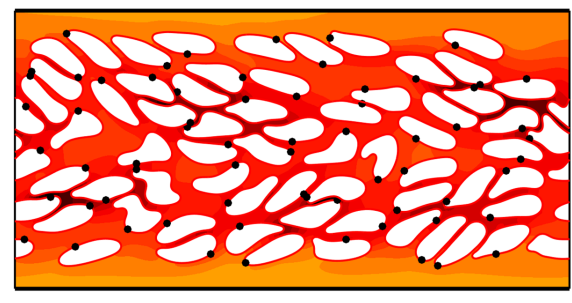

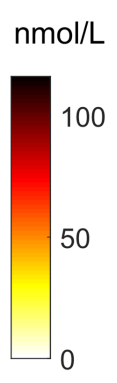

(b)

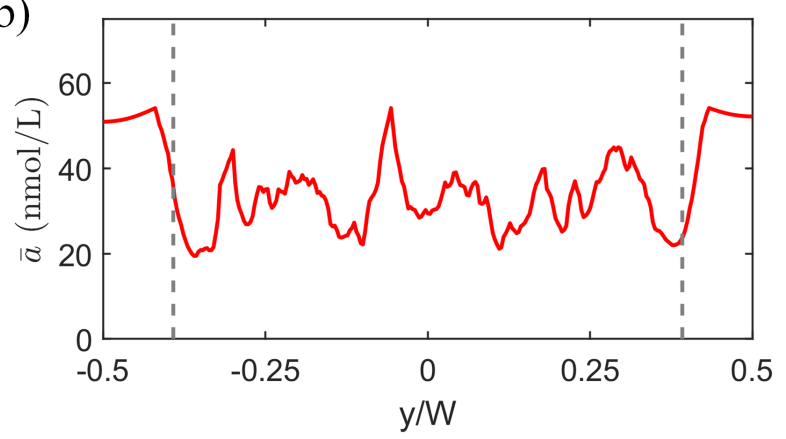

Figure 2: (a) RBC configuration and ATP distribution at $t=0.576 \mathrm{~s}$. The black dot on the cell membrane is a virtual tracer particle. (b) Average ATP concentration along the spanwise direction at the same time. The red solid line is the ATP concentration averaged along the flow direction (defined by equation 17). The gray dash lines denote the CFL boundary (the estimation of CFL follows the method provided in Ref. (42)). We see that there is more ATP at the CFL (two extreme positions of $y$ ) than in the core region. The capillary number is set as $C a=90$ and the viscosity ratio as $\lambda=6$.

Next, we investigate systematically how does flow (e.g. flow strength quantified by capillary number $\mathrm{Ca}$ ) affect the ATP release. Obviously, with the increase of capillary number, we expect an increase of ATP release per cell. Figure 3 a shows that the membrane shear stress increases linearly with the imposed pressure difference (or capillary number). In that figure we also show (gray dashed line) the critical membrane shear stress beyond which Px1 channel is activated (slightly beyond $\mathrm{Ca}=20$ ). 
Note that Figure 3a shows only the average over all cells, but this does not mean that below $C a=20$ all Px 1 channels are inactivated. For some cells, few Px1 reach the critical value, but this does not show up on the averaged value of shear stress (see the activation states of Px1 in Figure $4 a$ and b). We find here that the release via Px1 is predominant as compared to CFTR-upregulation. This is shown on Figure $3 \mathrm{~b}$ where the mean curvature change over the critical one (for CFTR activation) is significantly lower than unity. Note that while the average does not show CFTR effect, there are, however, few cells that are amply deformed leading to the participation of CFTR in the process of ATP release. In Figure $3 \mathrm{~b}$ (blue line) we show the maximum curvature change (over the critical one) as a function of $\mathrm{Ca}$. This maximum value exceeds unity for $\mathrm{Ca}>20$, meaning that there are few cells which significantly deform at a given moment, leading to CFTR-upregulation. Typical snapshots of the cells with CFTR activation are shown in Figure 4c-e. These cells are close to channel wall, and only a small portion of the membrane is CFTR-activated.

(a)

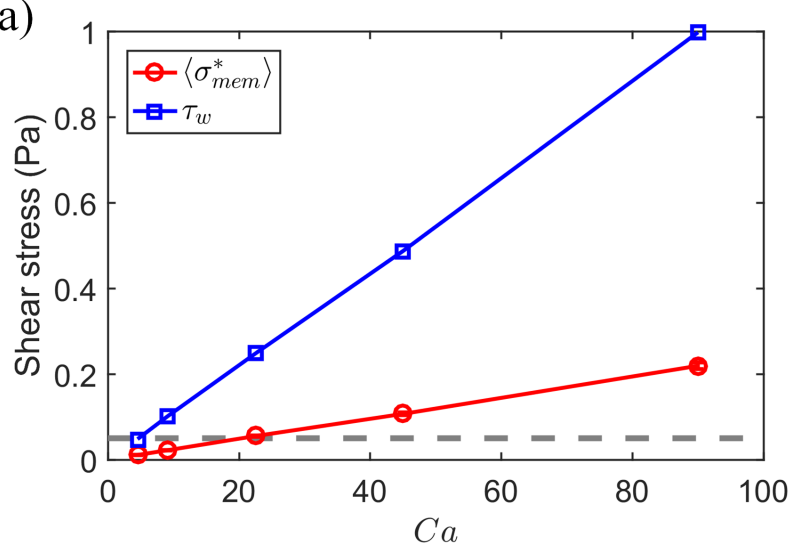

(c)

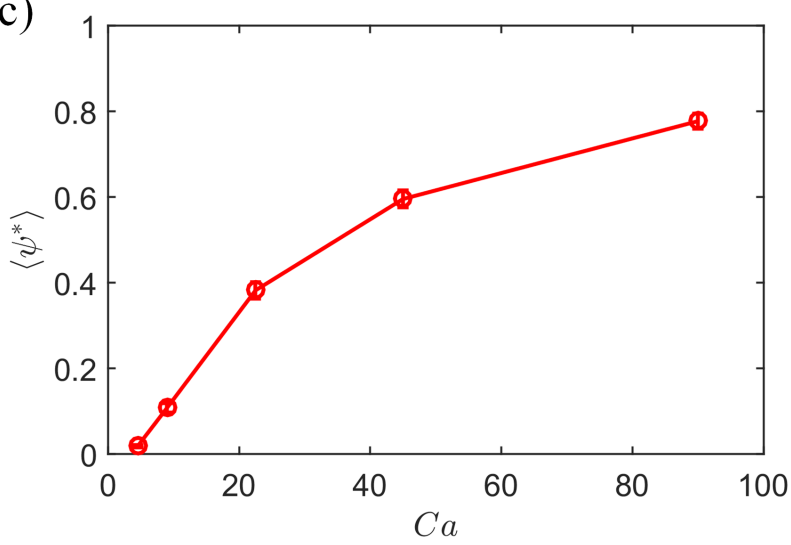

(b)

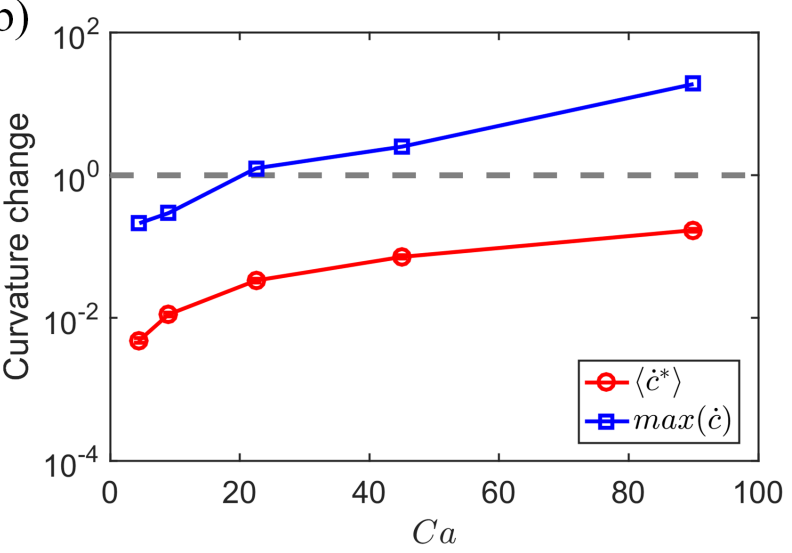

(d)

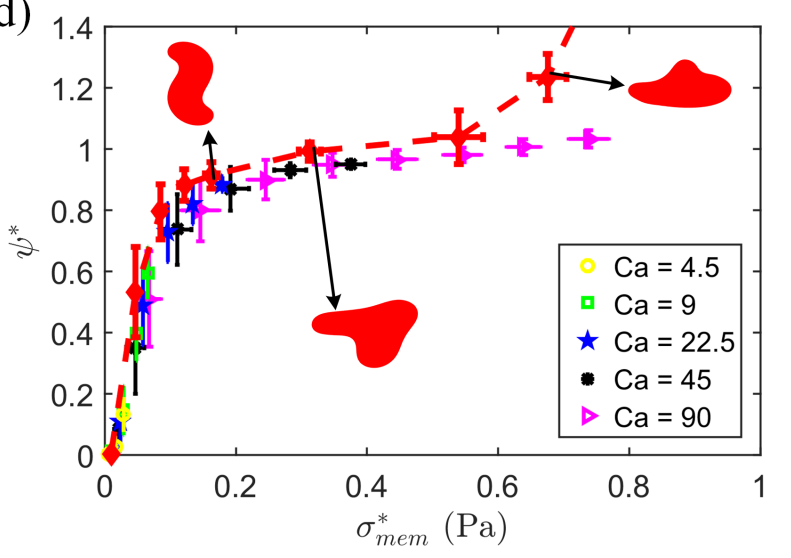

Figure 3: Average shear stress (both membrane shear stress $\left\langle\sigma_{m e m}^{*}\right\rangle$ and wall shear stress $\tau_{w}$ ) (a), curvature change rate over the critical one (b), and average ATP release level per cell $\left\langle\psi^{*}\right\rangle$ (c) versus capillary number with a viscosity ratio $\lambda=6$. The grey dashed lines in (a) and (b) correspond to the critical membrane shear stress for ATP release $\sigma_{c}$ and the critical curvature change $\dot{c}_{c}$ (dimensionless value in figure). The vertical error bars depict the SD. The average relation (over time and over all cells) between membrane shear stress and ATP release level is shown in (d). The error bars depict the SD of the membrane shear stress and ATP release. For that purpose we select small windows of shear stresses averaged over time, and each window corresponds to a subpopulation of cells. For example, cells in the central part release less ATP than cells at the periphery, since the shear stress there is small.The red dashed line in (d) represents the results of a single cell in simple shear flow. The shapes of the single cell at different membrane shear stresses are also shown. The data are obtained after the system has reached a permanent regime.

Now we examine the level of ATP release as a function of flow strength. The results are shown in Figure 3c. We report there the average ATP release rate per cell $\left\langle\psi^{*}\right\rangle$ as a function of $\mathrm{Ca}$. First we note that ATP release starts slightly below $\mathrm{Ca}=10$ (while, as stated above, the average critical value over all cells is $C a=20$; Figure 3a). In fact, a small proportion of cells reach the critical condition for ATP release for lower $\mathrm{Ca}$ but their weight in the average is small. Figure $3 \mathrm{c}$ shows that for small $\mathrm{Ca}$ the increase is quasilinear before a nonlinear behavior, leading to saturation, takes over. The saturation behavior is attributed to 
(a)

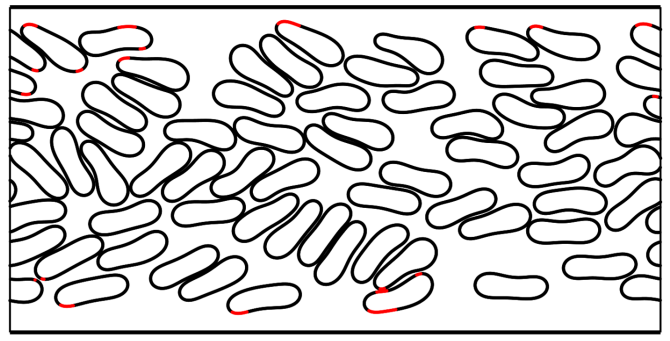

(c)

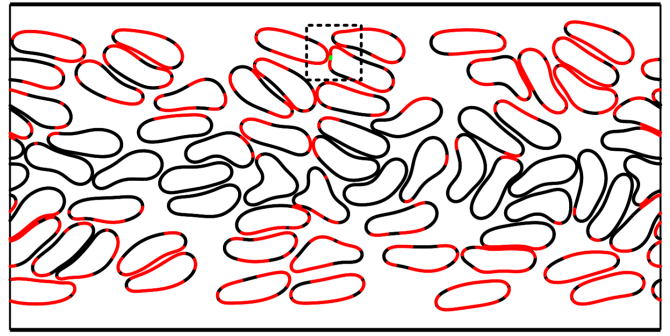

(b)

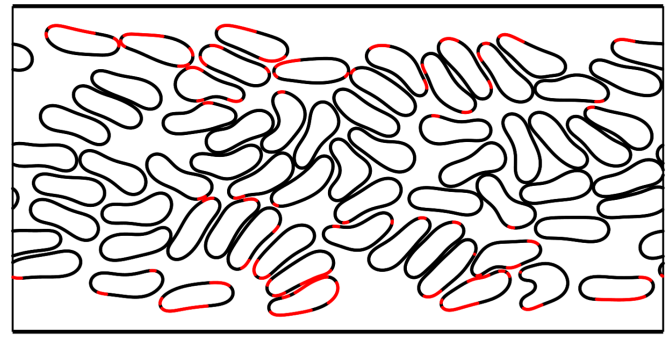

(d)

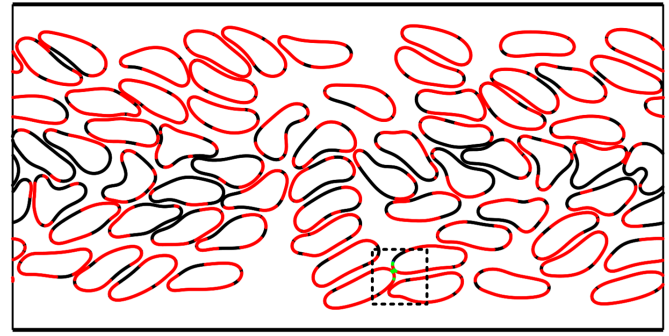

(e)

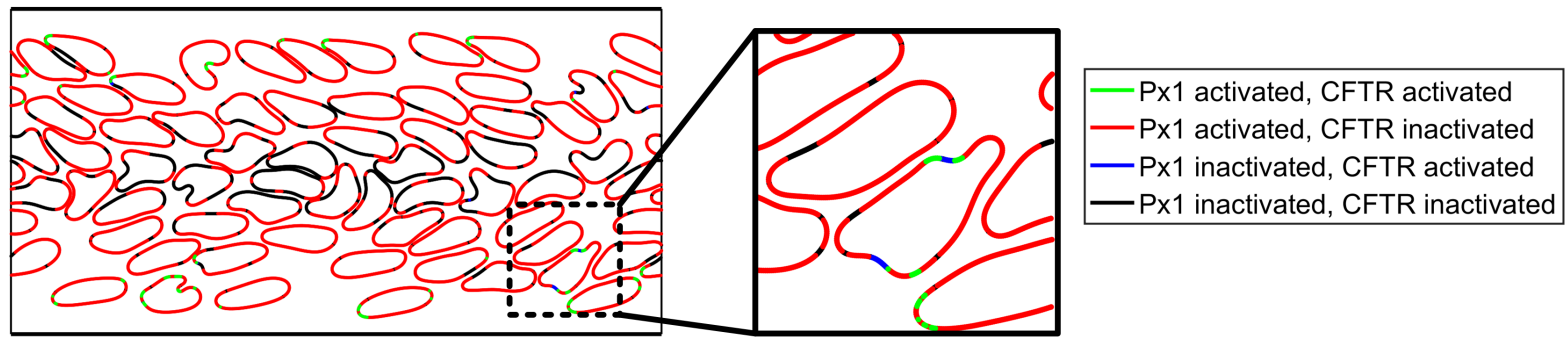

Figure 4: Px1 and CFTR activation states at $H t=46.0 \%$ : (a) $C a=4.5$ and $t=86.4 \mathrm{~s}$, (b) $C a=9$ and $t=43.2 \mathrm{~s}$, (c) $C a=22.5$ and $t=17.28 \mathrm{~s}$, (d) $C a=45$ and $t=2.88 \mathrm{~s}$, and (e) $C a=90$ and $t=1.44 \mathrm{~s}$. Four different activation states are identified: (i) both Px1 and CFTR inactivated (black), (ii) Px1 inactivated but CFTR activated (blue), (iii) Px1 activated but CFTR inactivated (red), and (iv) both Px1 and CFTR activated (green). Here $\sigma_{m e m}>\sigma_{c}$ means Px1 activation, and $\dot{c}>\dot{c}_{c}$ means CFTR activation. The dotted frames in (c) and (d) point to both Px1 and CFTR are activated. The partial zoom in (e) shows a cell that contains four different activation states.

the fact that, for high enough values of $\mathrm{Ca}$, many cells have already activated all Px1 channels (especially cells in the vicinity of walls where shear stress is high), so that increasing further $C a$ does affect significantly ATP release since most of channels had already been activated.

We have investigated systematically the effect of $H t$ (see Figure S2 in supplementary material). We have found that in a quite significant range of $H t$ the same general trend presented in Figure 3a-d holds. Let $\sigma_{m e m}^{*}$ denote the averaged shear stress over the membrane of a given cell (whereas $\left\langle\sigma_{m e m}^{*}\right\rangle$ is the average over all cells and over time), and $\psi^{*}$ the average (over a given cell at a given moment) ATP release per cell (not be confused with average over time and over all cells, denoted as $\left\langle\psi^{*}\right\rangle$ ). The results are shown on Figure 3d. The vertical and horizontal error bars depict the standard deviation (SD) of ATP release level and membrane shear stress, respectively. Interestingly enough, we find that all points fall reasonably on a master curve. A further support of this behavior comes from the analysis of ATP release in the case of a single cell (at a given moment), subject to different shear stresses. The corresponding result is shown in Figure $3 \mathrm{~d}$ by a dashed red line. Since the cell undergoes a complex dynamics (complex tumbling motion), we observed oscillations with time of membrane shear stress and ATP release level. The corresponding SD are shown by the vertical and horizontal error bars. For $\sigma_{m e m}^{*}<0.4 \mathrm{~Pa}$, the distribution of $\psi^{*}$ (corresponding to suspensions) and that corresponding to a single cell (dashed line) follow the same trend, and are quantitatively in a reasonable agreement. For larger shear stress, the single cell behavior deviates from the general trend by exhibiting a higher level of ATP release. As shown by the RBC shapes in Figure 3d, in the single cell case, the shape 
Gou et al.

deformation becomes quite large leading to the activation of the CFTR pathway, promoting higher ATP release. In contrast, for a concentrated suspension, the cell-cell interaction and crowding effect precludes cells to undergo large deformation so that ATP-release due to deformation remains small (albeit, as said before, a small proportion of cells can still release ATP due to deformation).

\section{Maximal ATP release for a given hematocrit}

In a recent work, we examined the volume fraction (hematocrit) for maximal cell transport in straight channels (43). It was found that the RBC volume fraction of healthy subjects is close to optimality for cell transport. We have naturally raised here a similar question whether there exists or not a given hematocrit for maximal ATP release per cell. For that purpose, we explored the effects of hematocrit in a range $H t \in[0.7 \%, 61.3 \%]$. The corresponding cell number $N_{c}$ is in the range [1,88]. The channel width is kept equal to that of the previous subsection (with $C n=0.133$ ).

The total ATP release level is defined as $\sum_{i}^{N} \psi^{*}$ and is shown in Figure 5a. It is obvious that higher hematocrit leads to higher total ATP release. For $C a \leq 9$, the total ATP release level shows a plateau, meaning that the increase of Ht does not cause additional release of ATP. The reason for this behavior will be explained latter.

(a)

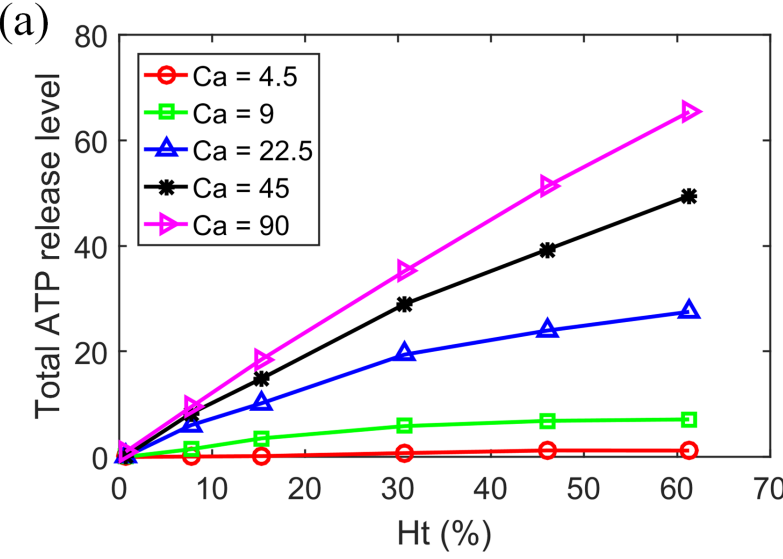

(c)

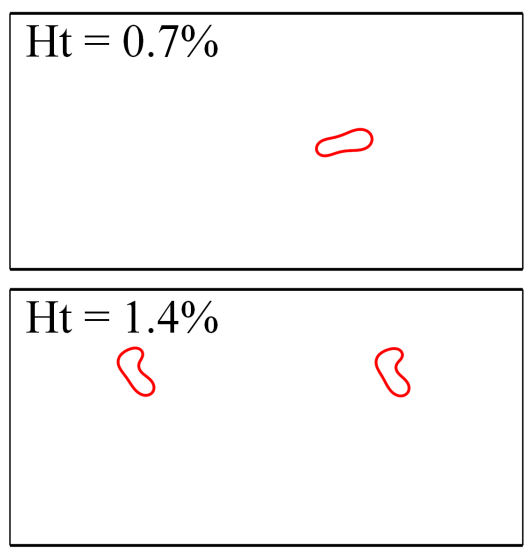

(b)

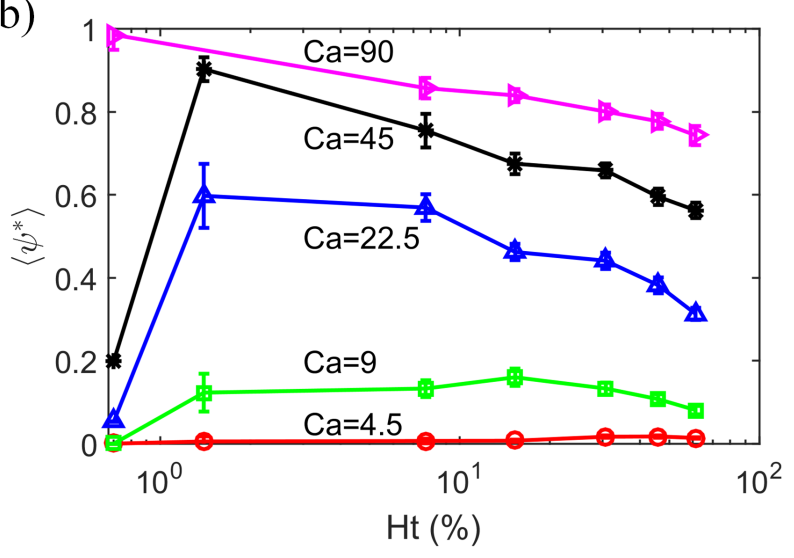

(d)

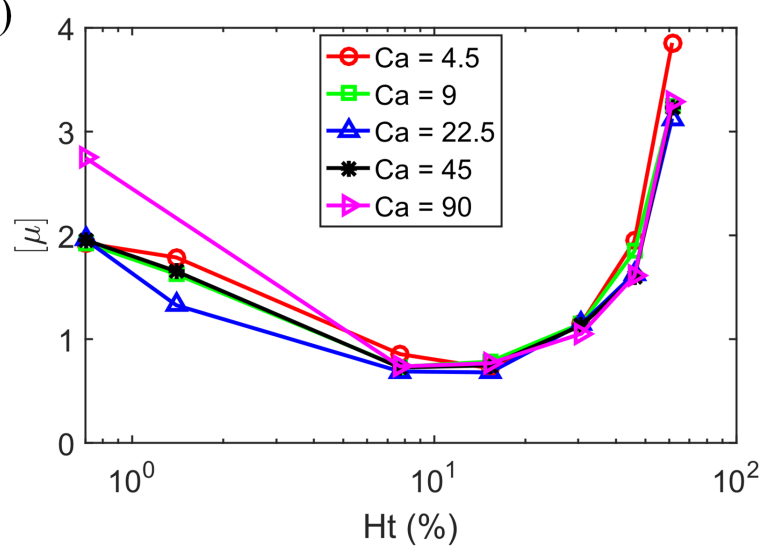

Figure 5: Total ATP release level (a), average ATP release level per cell (b), and intrinsic viscosity (d) at steady state. The vertical error bars in (b) depict the SD due to fluctuation over time. Snapshots of cell configuration at $C a=9$ are shown in (c). The confinement is fixed at $C n=0.133$.

A remarkable feature is exhibited when we analyze the average ATP release level per cell $\left\langle\psi^{*}\right\rangle$ as a function of $H t($ Figure $5 b$ ), instead of total ATP release. This quantity shows a non-trivial non-monotonic behavior in a wide range of flow strength (or $\mathrm{Ca}$ ). The error bars in Figure 5b show the SD (due to fluctuations over time). It should be noted that after the system reaches permanent regime, the presented SD with respect to time is small. In supplementary materials (see Figure S3) we show other statistics, where we exhibit heterogeneities between cells, highlighting the fact that different cells (depending, for example, on their lateral position in the channel) release different ATP levels due to the difference in shear stress. The heterogeneities 
among cells due to their lateral position in channel are shown in Figure 6 as well. For all cases with $C a<90$ we find a maximal ATP release level for a given value of hematocrit. Except for the smallest explored $C a(C a=4.5)$, the critical hematocrit lies in the range $H t=0-20 \%$, which corresponds to typical values of hematocrit in microcirculation (44). This means that adding further cells beyond this hematocrit is not beneficial for ATP release. The main reason behinds the existence of maximal ATP release at a given $H t$ is the following. At low hematocrit, cells tend to be centered in the channel due to a hydrodynamic lift force $(5,45,46)$; the RBCs form a single centered file (see the upper panel of Figure 5c). Since at the center the shear stress is the smallest, most Px1 channels are inactivated, resulting in a low ATP level per cell. On increasing further $H t$ the central RBCs file splits into several files pushing some cells towards periphery where the shear stress is higher (see the lower panel of Figure $5 \mathrm{c}$ ). It should be noted that the number of cells in a central file is affected by the confinement (in the next section, we will show stable central files with many cells for different confinement). For the present confinement $(C n=0.133)$, however, only a single cell survives at the center as a stable configuration. Adding a second cell leads to off-center position for both cells (and thus higher shear stress and higher ATP; Figure 5c, lower panel). The expelling of cells from center results in an increase of ATP release level per cell. In addition, cells in regions of high shear stress undergo higher deformations provoking CFTR ATP-upregulation, reinforcing thus further ATP release. Significantly higher $H t$ acts against ATP release. The first reason for this is due to the decrease of CFL. As shown before (Figure 2b) CFL contributes significantly to ATP release, and its reduction leads to a decline of ATP release. The second effect is due to the fact that in a concentrated suspension the flow felt by a given cell is screened by surrounding cells, resulting in a lower shear stress felt by the cells, and thus in a lower activation of Px 1. The level of ATP release per cell consequently decreases beyond a certain $\mathrm{Ht}$ (see the comparison between Figure $6 \mathrm{c}$ and d). All together the ATP release should show a maximum at a given $\mathrm{Ht}$.

To further dig into this behavior, we have analyzed another property of blood flow, namely the intrinsic viscosity. Figure $5 \mathrm{~d}$ shows the intrinsic viscosity $[\mu]$. This quantity is more appropriate than the effective viscosity since it represents the contribution of each cell to viscosity (where the solvent viscosity is subtracted). A reduced viscosity at a given $\mathrm{Ht}$ means also a higher flow rate, and thus a higher oxygen transport. Interestingly, the intrinsic viscosity shows a minimum at a hematocrit close to that corresponding to the maximal ATP release. This maximal ATP release seems to coincide with an efficient oxygen transport as well.

Actually, the results of Figure $5 \mathrm{~b}$ call for two remarks. (i) The $H t$ for maximal ATP release is about $40 \%$ for small flow strength $(C a=4.5)$, whereas for higher $C a$ it lies in the range $H t=0 \sim 20 \%$. (ii) For extreme values of $C a(C a=90)$ there is a monotonic behavior (at least for the explored channel width in Figure 5b). The first effect can be understood as follows. As discussed above, at small $H t$ cells gather together in the center line. Increasing $H t$ until the central line becomes crowded with RBCs, will cause expelling of some cells from center to nearby peripheral locations (see the lower panel of Figure 5c). However, since the flow strength is small, a sufficient number of cells is needed so that some of them will occupy far enough positions out of center, where shear stress is sufficiently large to trigger ATP release (see the comparison between Figure 6a and b). The smaller $\mathrm{Ca}$ the larger $\mathrm{Ht}$ must be in order to find enough RBCs in high enough shear stress regions. This explain why small flow strengths lead to a higher value of $H t$ corresponding to maximal ATP release.

The second phenomenon (suppression of critical hematocrit for $\mathrm{Ca}=90$ ) has a completely different origin. Indeed, while usually it is commonly found in literature that in a Poiseuille flow cells tend to migrate to the center line, this is not always true. Here, we find that there exists a critical flow strength where the central position becomes unstable, and a cell will migrate outwards. This type of solution is akin to the slipper solution (a single cell becomes off-centered) reported in (47, 48). In particular, it was observed in (49) that for the viscosity contrast chosen here, and at large enough $\mathrm{Ca}$ (about 100) cells will migrate indefinitely to the periphery. In that work, however, no lateral wall was taken into account (unbounded parabolic flow), and the cell migrated outwards without bound. In the presence of walls, Figure 7 a shows the final shape and positions as a function of $C a$. For $C a=90$ the RBC is off-centered at position about $0.2 \mathrm{~W}$. For a small $H t, \mathrm{RBCs}$ will organize in one or two files outside center and release high enough ATP (see Figure 7b). Increasing $H t$ causes crowding of these peripheral files and some RBCs are expelled towards center, where the shear rate is smaller, resulting in a decrease of ATP release. The ATP release should exhibit thus a monotonic decreasing behavior with $H t$.

The lateral migration of cell at small $H t$ has an influence on intrinsic viscosity as well. As shown in Figure 7 a, the single cell (i.e. $H t=0.7 \%$ ) stays close to the center of the channel for $C a \leq 45$. For this reason, the contribution to intrinsic viscosity is independent of $\mathrm{Ca}$ (see Figure 5d). When $\mathrm{Ca}=90$, the cell migrates to an off-centered position, which leads to higher intrinsic viscosity.

Figure 7c quantifies the above results. It shows the proportion of cells which are activated for different $H t$ and $C a$. We define the probability of a cell that releases ATP as

$$
\phi_{\mathrm{ATP}}=\frac{N_{\mathrm{ATP}}}{N_{c}},
$$

where $N_{\text {ATP }}$ is the number of cells that satisfy $\psi^{*}>0$ (i.e. cells for which Px1 channels are activated). For high $C a$ most cells 
(a)

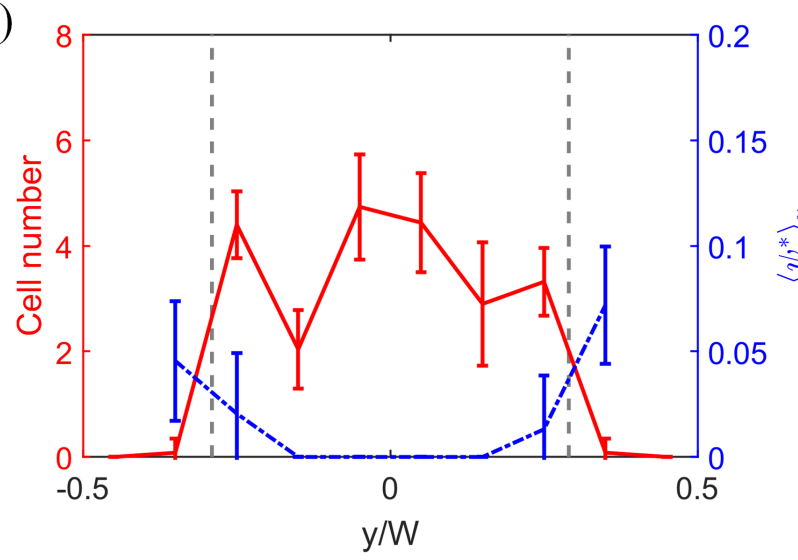

(c)

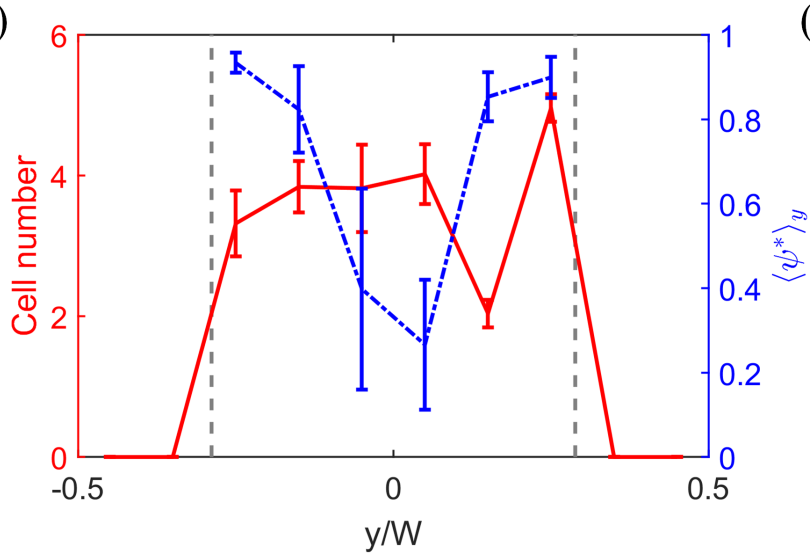

(b)



(d)

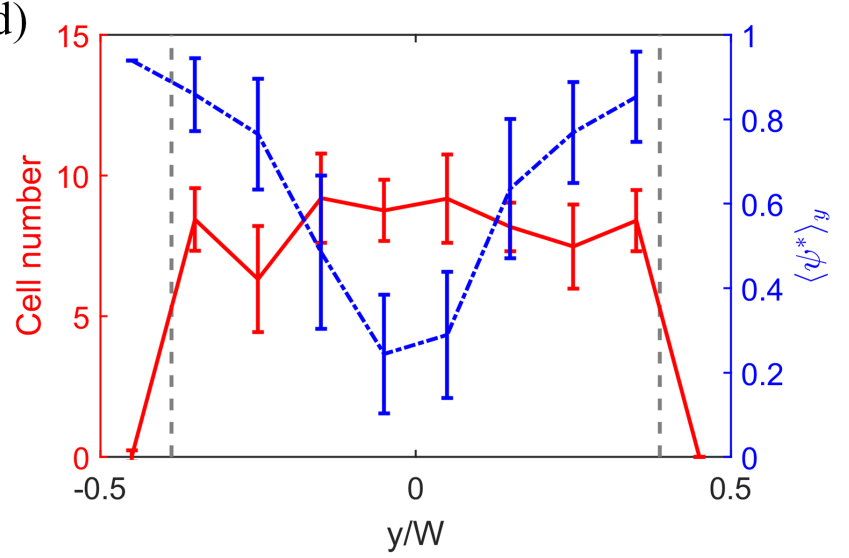

Figure 6: The cell number (red solid line) and ATP release level (blue dash-dotted line) along the spanwise direction. The gray dashed lines indicate the CFL boundary. The left column ((a) and (c)) refers to $H t=15.3 \%$ and the right column ((b) and (d)) to $H t=46.0 \%$. The upper row ((a) and (b)) refers to $C a=4.5$ and the lower row ((c) and (d)) to $C a=45$. The channel is divided into 10 sections along the spanwise direction. In each section, the cell number and ATP release level of each cell are calculated within a long enough time window. For the cell number, the mean values are averaged over time, and the error bars refer to the SD with respect to time. For the ATP release level, the mean values are averaged over all cells present in a section, and the error bars refer to the SD with respect to these cells.

are activated, whereas for smaller $C a$ activation gradually increases with $H t$, since increasing $H t$ results progressively in cells expelled outwards where shear stress is strong.

At medium and high capillary number, the value of $\phi_{\text {ATP }}$ is barely affected by $H t$, (Figure $7 \mathrm{c}$, blue, black and magenta lines) since most Px 1 are already activated at those values of $C a$. For lower $C a$ (green line in Figure 7c) the ATP declines by increasing $\mathrm{Ht}$, due to the aforementioned screening effect. For the lowest capillary number $(\mathrm{Ca}=4.5)$, the cells are, for small $H t$, close to the center line where shear stress is small enough so that a very small amount of Px1 is activated, resulting in a low ATP release. Increasing hematocrit causes cells to spread out in the spanwise direction (see Figure $6 \mathrm{a}, \mathrm{b}$ and Figure $7 \mathrm{~d}, \mathrm{e}$ ). This leads to an increase of ATP release, and then to saturation due to screening effects.

To confirm the non-monotonic behavior of average ATP release level per cell, a systematic analysis in 3D is conducted. The channel width is $W=5 R_{0}(C n=0.4)$, and the capillary number is $C a=52$. As shown in Figure 8a, a maximum ATP release level per cell (red solid line) is found between $H t=0-10 \%$, in qualitative agreement with the results in 2D. The reason for this non-monotonic behavior is the same as described above. At low hematocrit, cells are concentrated around the center of channel, experiencing a smaller shear stress (see Figure 8 b). With the increase of hematocrit, there is an interplay between the lateral migration which pushes cells to high shear stress area, and the screening effect which decreases shear stress (see Figure 8c and d). As a result, the average membrane shear stress shows a non-monotonic behavior (see the blue dashed line in Figure 8a), and so does the ATP release level per cell. 
(a)

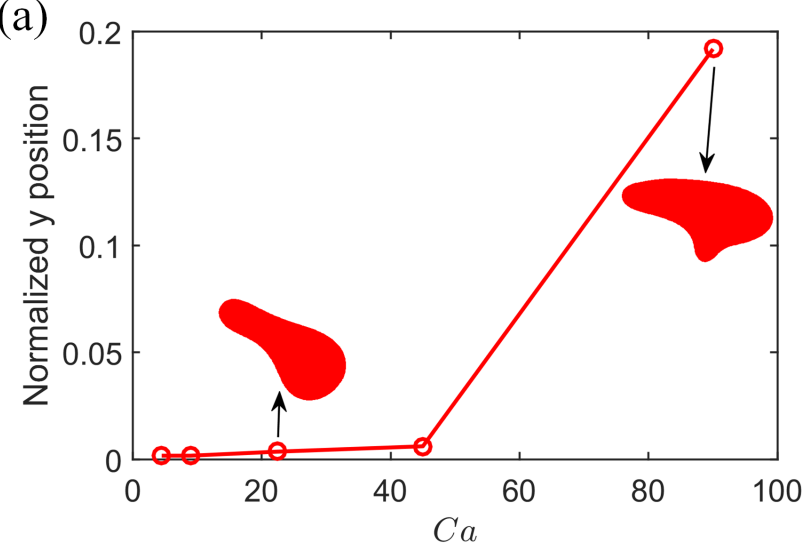

(c)

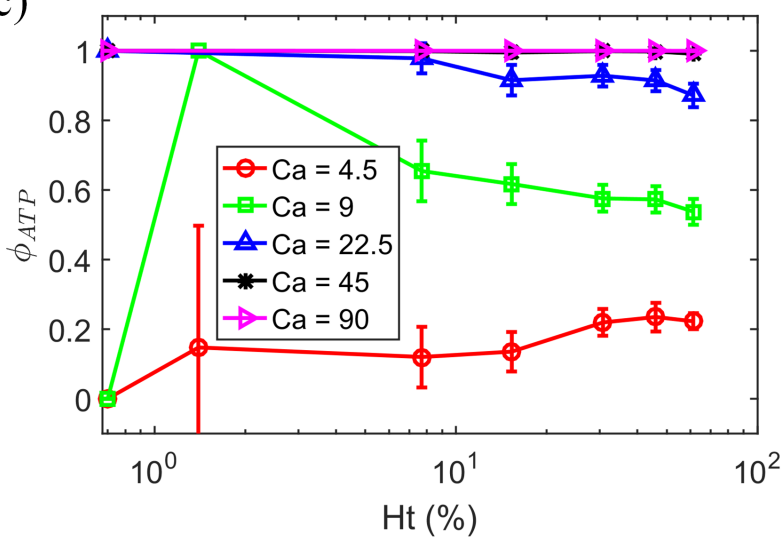

(d)

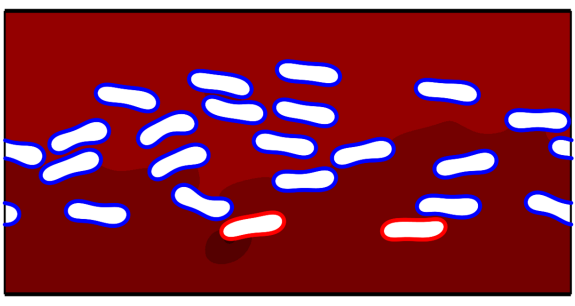

$\mathrm{nmol} / \mathrm{L}(\mathrm{e})$

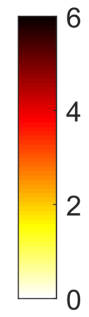

(b)
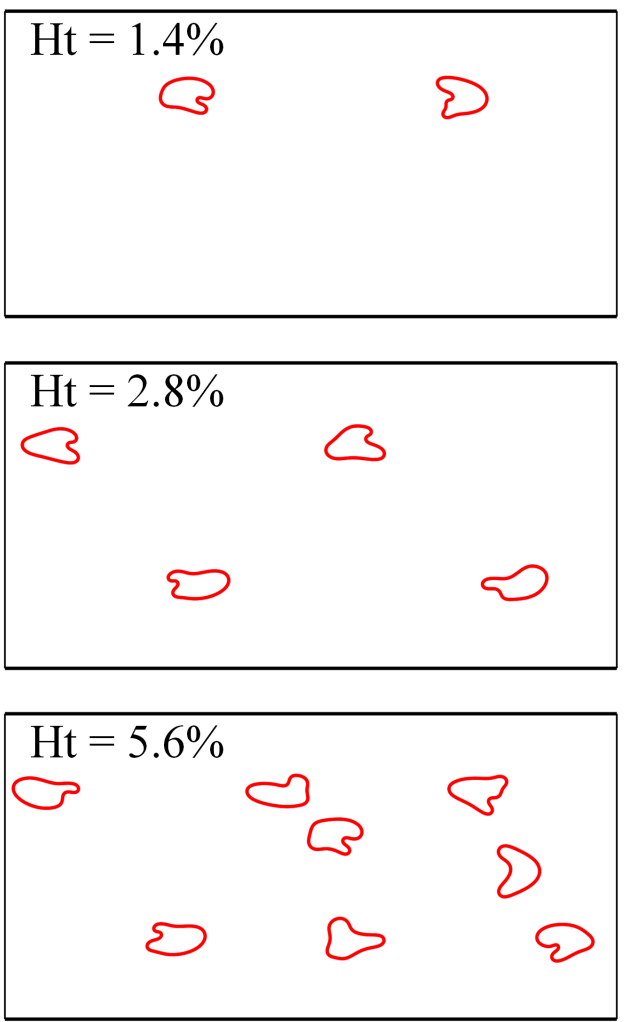

$\mathrm{nmol} / \mathrm{L}$

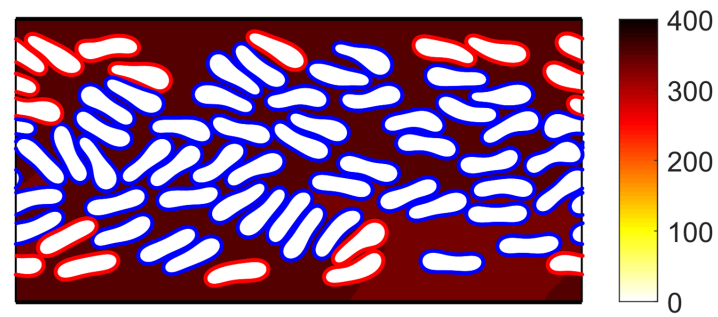

Figure 7: (a) Normalized $y$ position for $H t=0.7 \%$ (single cell condition). (b) Snapshots of cell configuration at low $H t$ and $C a=90$. (c) Probability of cell that release ATP $\phi_{\text {ATP }}$ (the definition of $\phi_{\text {ATP }}$ is given in equation 18). The error bars depict the $\mathrm{SD}$ with respect to time. RBC configuration and ATP distribution at $C a=4.5$ are shown in (d) for $H t=15.3 \%$ and $t=14.4$ $\mathrm{s}$, and (e) for $H t=46.0 \%$ and $t=86.4 \mathrm{~s}$. The red and blue lines represent RBCs that release ATP and do not release ATP, respectively.

\section{Effects of confinement}

When flowing in microcirculation, RBCs experience a cascade of branching vessels, from arterioles to capillaries, and finally to venules. These vessels have different diameters, ranging from $\sim 10 \mu \mathrm{m}$ to $\sim 100 \mu \mathrm{m}$. Previous studies have shown that the change of vessel diameter can affect cell dynamics, rheology (43,50-52), as well as ATP release (15). It is therefore important to explore other values of confinements. The capillary number is fixed at a given value for different confinements. This means that the local shear rate of the undisturbed flow falls into the same range for different channel widths. Then it is reasonable to compare these results as shown in Figure 9. Recall that the above study was dedicated to $C n=0.133$ (corresponding to vessel diameter of about $45 \mu \mathrm{m}$, lying in the range of arterioles). Increasing progressively confinement still shows the same 
(a)

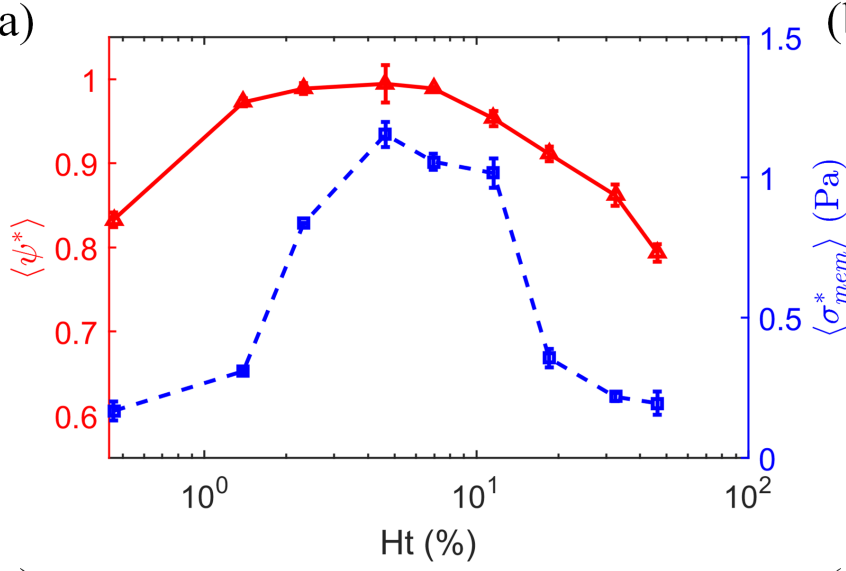

(c)

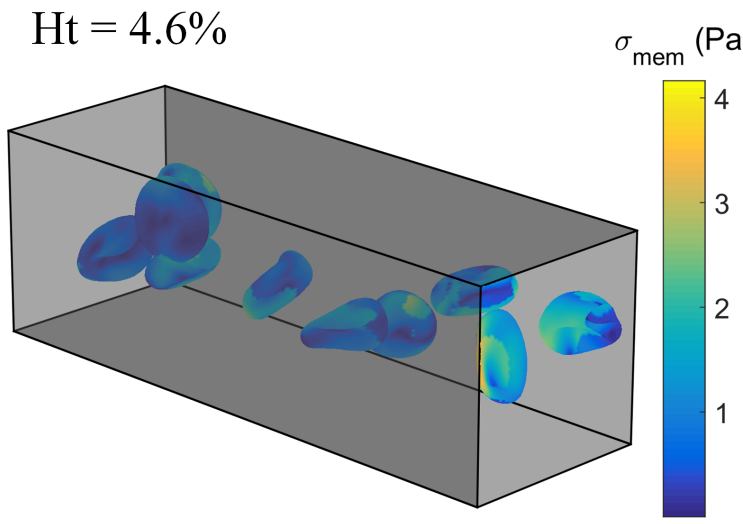

(b)

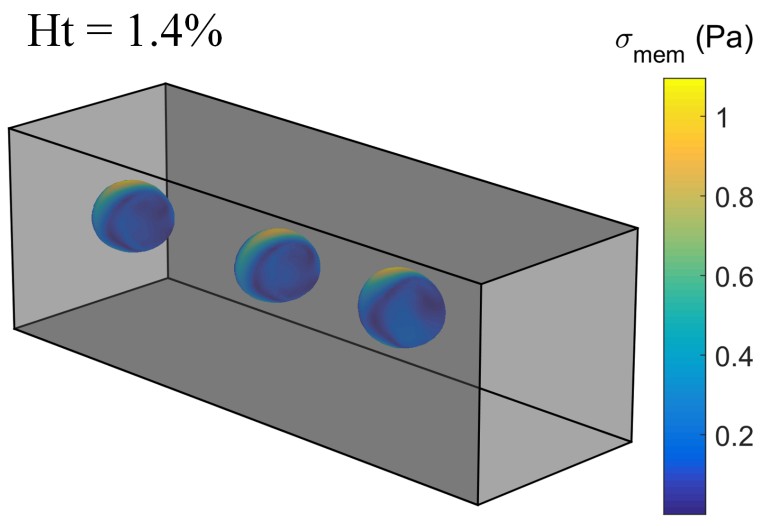

(d) $\mathrm{Ht}=18.5 \%$

$\sigma_{\text {mem }}(\mathrm{Pa})$

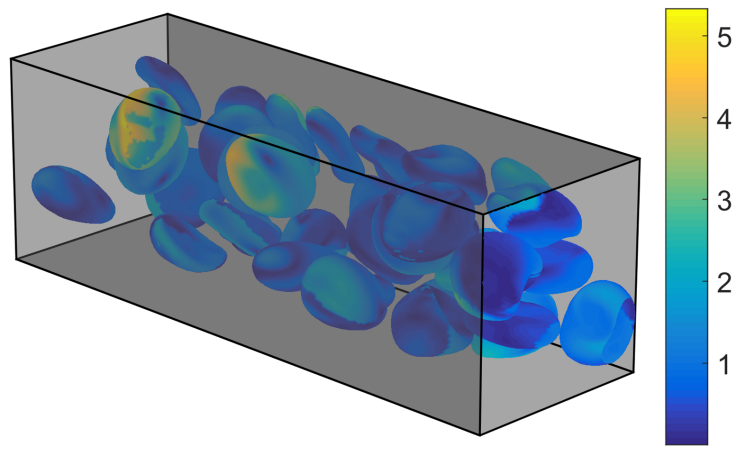

Figure 8: (a) Average ATP release level per cell $\left\langle\psi^{*}\right\rangle$ (red solid line) and average membrane shear stress $\left\langle\sigma_{\text {mem }}^{*}\right\rangle$ (blue dashed line) at $\mathrm{Ca}=52$. The error bars depict the SD over time. (b), (c), and (d) show snapshots of cell configuration and membrane shear stress at different hematocrits.

qualitative behavior as before, in that the ATP release level exhibits a maximum (Figure 9a). Two observations can be made: (i) the hematocrit at which ATP is maximal, $H t^{*}$ increases by decreasing vessel diameter, (ii) the absolute ATP level decreases upon decreasing the diameter. Figure 9a shows the results for $C n=0.16$ and $C n=0.2$, and compared to the case $C n=0.133$ (studied above). The fact that $H t^{*}$ increases by decreasing vessel diameter is traced back to the fact that cells are more stabilized in the center line (where shear stress is small) due to the influence of walls which exerts a higher hydrodynamic lift force when vessel diameter is small (see also Figure S4 in supplementary materials). Upon increasing $H t$, ATP release increases due to cells being expelled from center (due to crowding at the center), where shear stress is lower. Here again, a large enough $H t$ leads to screening effects which leads to a collapse of ATP release.

Investigating smaller diameters leads to a remarkable feature in capillaries. Figure $9 \mathrm{~b}$ shows that in arterioles (blue line) the ATP release is higher than in post capillary venules (green line). However, when reaching small enough vessel diameters (range of capillaries) the ATP release is boosted, as shown in Figure $9 \mathrm{~b}$ (red line). The probability distribution of ATP release level of cells is given in supplementary material (see Figure S5). For $C n=0.8$, corresponding to vessel diameter of $7.5 \mu \mathrm{m}$ (capillaries range), we see in Figure $9 \mathrm{~b}$ that the ATP release level is close to $70 \%$, significantly above other sites of microvasculature. In capillaries the maximal ATP release per cell is obtained at very small $\mathrm{Ht}$ (typically for a single cell in the channel, corresponding to few percent's of $\mathrm{Ht}$ ). The prevalence of ATP release in capillaries can be explained as follows. While the cells remain in the center (due to strong confinement), their periphery is always close enough to channel walls where shear stress is the highest (see Figure 9c).

The fact that ATP is boosted in capillaries is a priori surprising, owing to the absence of smooth muscle cells. Of all blood vessels, capillaries are most numerous and most closely associated with muscle fibers. This raises the question of whether capillary ECs can be stimulated to trigger a response that travels upstream to influence the diameter and blood flow through parent arterioles. ATP binding to P2Y receptors is a candidate for EC hyperpolarization leading to electrical stimulus that 
(a)

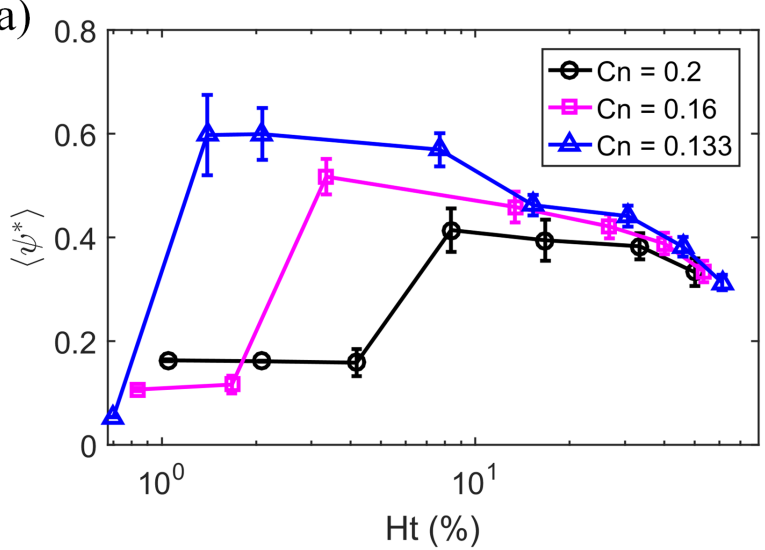

(c)

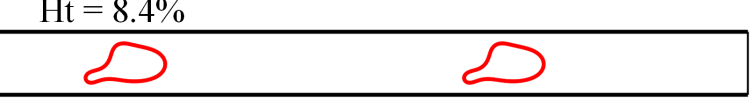

(d)

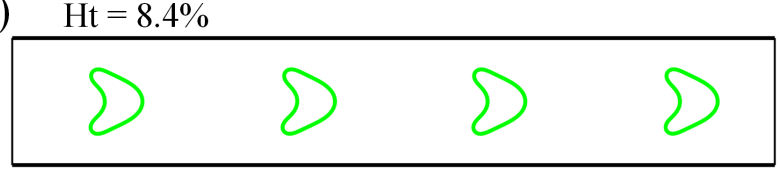

(b)
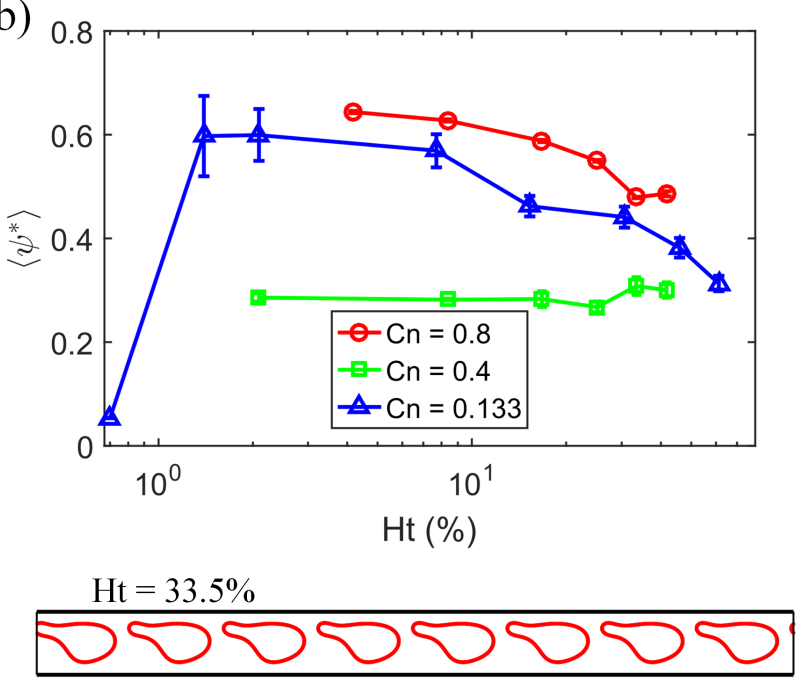

$\mathrm{Ht}=33.5 \%$

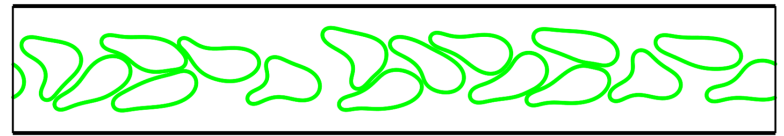

Figure 9: (a) and (b) show average ATP release level per cell affected by confinement at $C a=22.5$. The error bars depict the SD over time. Note that the scales of vertical axis are different. Snapshots of cell configuration are shown in (c) for $C n=0.8$ and (d) for $C n=0.4$, using corresponding colors as the curves in (b).

can travel over several millimeters in arterial networks (53). There are several evidences of arterial vasodilation caused by electrical stimuli from capillaries. Vasodilation of arterial trees would thus be coordinated throughout the vascular network, where capillaries play an active role.

\section{CONCLUSION}

In this work, we have adopted the ATP release model proposed by Zhang et al (15) and extended it to multi-cell system. By using a lattice-Boltzmann solver, we have numerically investigated the ATP release by RBCs in Poiseuille flow, and its relationship to flow strength, hematocrit and confinement.

We first studied the channel width of $45 \mu \mathrm{m}$, approximately the size of arterioles and venules. ATP released by RBCs has been shown ex vivo to critically affect calcium signaling (41). How ATP is distributed within a vessel is not an easy experimental task. In this work, we were able to demonstrate the distribution of ATP concentration in the channel, and showed that the real ATP concentration was higher at the CFL. Since the CFL is close to the ECs, the increase of ATP concentration there would facilitates the reaction with EC in the process of $\left[\mathrm{Ca}^{2+}\right]$ signaling (41). ATP release is found to be correlated with flow strength, implying that ATP release was more significant in arterioles than in venules.

The ATP release was mainly due to Px1 channel in the range of flow strengths explored so far. The CFTR was barely activated because large deformation was inhibited due to cell-cell interaction. Actually, the membrane deformation is affected by the mechanical properties of the cells. The softening or hardening of cell membrane due to certain diseases (e.g. cancer (54) and sickle cell anemia $(55,56))$ could result in abnormal ATP release by RBCs.

When considering the effect of hematocrit, we observed a given hematocrit for yielding a maximal ATP release level per cell, at which the intrinsic viscosity reached a minimum value as well, suggesting a compromise between ATP release and a minimal blood dissipation. The specific hematocrit found here is consistent with values in microcirculation. Whether or not the maximal ATP release corresponds to an optimal physiological value is presently not clear and needs further investigations.

We found that the variation of hematocrit greatly affects RBC configurations and ATP release in more confined channels. Specifically, we found that ATP release is boosted in capillaries, despite the absence of smooth muscle cells there. This suggests that capillaries, the most abundant sites in the microvasculature, may play a central role in blood flow regulation 
through upstream communication with parent arterioles. ATP is known to react with P2Y endothelial receptors resulting in EC hyperpolarization of the capillary endothelium. These electrical signals may travel upstream fast enough across the entire capillary bed into the arteriolar tree to direct the distribution of $\mathrm{O}_{2}$ supply to where it is needed (57).

Extracellular ATP is also involved in vascular pathologies such as atherosclerosis $(58,59)$. The formation of atherosclerotic plaques is often found at sites with low wall shear stress. The present work suggests that ATP release of RBCs is reduced at low shear stress sites, leading to insufficient extracellular ATP at these sites. As a result, the $\left[\mathrm{Ca}^{2+}\right]$ signaling in EC is affected, and so is the synthesis of NO, which acts as a key inhibitor of atherogenesis (60). The deficiency of vascular NO is known to contributes to the development of atherosclerosis (61).

An interesting future task is the study of ATP release in complex vascular networks including capillaries and arterioles. It has been shown recently (15) that bifurcations can significantly enhance ATP release, due to ample deformation of RBCs at the bifurcation points. The study of ATP patterns in realistic networks is the next natural step, before tackling biochemical cascades such as (i) calcium signaling (currently under investigation), (ii) EC electrical propagation from capillaries to parent arterioles. These numerical studies, which are hoped to be developed in a concerted fashion with experiments (using our recent microvasculature on chip (62)), are expected to give new insights into the imbricated nature of biochemical signaling in the microvasculature. Moreover, we have obtained a qualitative agreement between 2D and 3D simulations.

\section{AUTHOR CONTRIBUTIONS}

Z.G. has contributed to the code and performed simulations and analysis of data. H.Z. has developed the ATP model and the corresponding code. C.M. has designed the research topic and plans. M.A. has performed simulations and analysis of data. All the authors have contributed to the article writing and interpretations.

\section{ACKNOWLEDGMENTS}

This work was supported by Centre National d'Etudes Spatiales (4500061585); and French-German University Programme "Living Fluids" (CFDA-Q1-14). We thank Centre National d'Etudes Spatiales for allowing having access to experimental data. The simulations were performed on the Cactus cluster of the CIMENT infrastructure, which is supported by the Rhône-Alpes region (GrantNo.CPER07 13 CIRA). We thank Jens Harting for help on simulations.

\section{SUPPORTING MATERIAL}

Supporting material can be found online at https://doi.org/10.1016/j.bpj.2021.09.025.

\section{REFERENCES}

1. Schwiebert, E. M., and A. Zsembery, 2003. Extracellular ATP as a signaling molecule for epithelial cells. Biochimica et Biophysica Acta (BBA) - Biomembranes 1615:7-32.

2. Furchgott, R. F., 1984. The role of endothelium in the responses of vascular smooth muscle to drugs. Annu Rev Pharmacol Toxicol 24:175-97. https://www.ncbi.nlm.nih.gov/pubmed/6203480.

3. Burnstock, G., and C. Kennedy, 1986. A dual function for adenosine 5'-triphosphate in the regulation of vascular tone. Excitatory cotransmitter with noradrenaline from perivascular nerves and locally released inhibitory intravascular agent. Circ Res 58:319-30. https://www.ncbi.nlm.nih.gov/pubmed/3013455.

4. Brooks, D. E., J. W. Goodwin, and G. V. F. Seaman, 1970. Interactions among erythrocytes under shear. Journal of Applied Physiology 28.

5. Zhang, H., 2018. Simulation of blood microcirculation and its coupling to biochemical signaling. Thesis.

6. Sprague, R. S., M. L. Ellsworth, A. H. Stephenson, M. E. Kleinhenz, and A. J. Lonigro, 1998. Deformation-induced ATP release from red blood cells requires CFTR activity. Am J Physiol 275:H1726-32. https://www.ncbi.nlm.nih.gov/ pubmed/9815080.

7. Carroll, J., M. Raththagala, W. Subasinghe, S. Baguzis, T. D'Amico Oblak, P. Root, and D. Spence, 2006. An altered oxidant defense system in red blood cells affects their ability to release nitric oxide-stimulating ATP. Mol Biosyst 2:305-11. https://www.ncbi.nlm.nih.gov/pubmed/16880949. 
8. Subasinghe, W., and D. M. Spence, 2008. Simultaneous determination of cell aging and ATP release from erythrocytes and its implications in type 2 diabetes. Anal Chim Acta 618:227-33. https://www.ncbi.nlm.nih.gov/pubmed/ 18513544.

9. Sprague, R. S., M. L. Ellsworth, A. H. Stephenson, and A. J. Lonigro, 1996. ATP: the red blood cell link to NO and local control of the pulmonary circulation. Am J Physiol 271:H2717-2722.

10. Fischer, D. J., N. J. Torrence, R. J. Sprung, and D. M. Spence, 2003. Determination of erythrocyte deformability and its correlation to cellular ATP release using microbore tubing with diameters that approximate resistance vessels in vivo. Analyst 128:1163-8. https://www.ncbi.nlm.nih.gov/pubmed/14529024.

11. Wan, J., W. D. Ristenpart, and H. A. Stone, 2008. Dynamics of shear-induced ATP release from red blood cells. Proc Natl Acad Sci U S A 105:16432-7. https://www.ncbi.nlm.nih.gov/pubmed/18922780.

12. Locovei, S., L. Bao, and G. Dahl, 2006. Pannexin 1 in erythrocytes: Function without a gap. Proc Natl Acad Sci U S A 103:7655-7659.

13. Chasan, B., N. A. Geisse, K. Pedatella, D. G. Wooster, M. Teintze, M. D. Carattino, W. H. Goldmann, and H. F. Cantiello, 2002. Evidence for direct interaction between actin and the cystic fibrosis transmembrane conductance regulator. Eur Biophys J 30:617-24. https://www.ncbi.nlm.nih.gov/pubmed/11908853.

14. Forsyth, A. M., J. Wan, P. D. Owrutsky, M. Abkarian, and H. A. Stone, 2011. Multiscale approach to link red blood cell dynamics, shear viscosity, and ATP release. Proc Natl Acad Sci U S A 108:10986-91. https://www.ncbi.nlm.nih. gov/pubmed/21690355.

15. Zhang, H., Z. Shen, B. Hogan, A. I. Barakat, and C. Misbah, 2018. ATP Release by Red Blood Cells under Flow: Model and Simulations. Biophys J 115:2218-2229. https://www.ncbi.nlm.nih.gov/pubmed/30447988.

16. Albrecht, K. H., P. Gaehtgens, A. Pries, and M. Heuser, 1979. The Fahraeus effect in narrow capillaries (i.d. 3.3 to 11.0 micron). Microvasc Res 18:33-47. https://www.ncbi.nlm.nih.gov/pubmed/481244.

17. Pries, A. R., K. Ley, and P. Gaehtgens, 1986. Generalization of the Fahraeus principle for microvessel networks. Am J Physiol 251:H1324-32. https://www.ncbi.nlm.nih.gov/pubmed/3789184.

18. Goldsmith, H. L., G. R. Cokelet, and P. Gaehtgens, 1989. Robin Fahraeus: evolution of his concepts in cardiovascular physiology. Am J Physiol 257:H1005-15. https://www.ncbi.nlm.nih.gov/pubmed/2675631.

19. Abkarian, M., M. Faivre, R. Horton, K. Smistrup, C. A. Best-Popescu, and H. A. Stone, 2008. Cellular-scale hydrodynamics. Biomed Mater 3:034011. https://www.ncbi.nlm.nih.gov/pubmed/18765900.

20. Shen, Z. Y., A. Farutin, M. Thiebaud, and C. Misbah, 2017. Interaction and rheology of vesicle suspensions in confined shear flow. Physical Review Fluids 2. <GotoISI>://WOS : 000412438800001.

21. Krüger, T., F. Varnik, and D. Raabe, 2011. Efficient and accurate simulations of deformable particles immersed in a fluid using a combined immersed boundary lattice Boltzmann finite element method. Computers \& Mathematics with Applications 61:3485-3505. https://www.sciencedirect.com/science/article/pii/s0898122110002476, mesoscopic Methods for Engineering and Science — Proceedings of ICMMES-09.

22. Krüger, T., S. Frijters, F. Günther, B. Kaoui, and J. Harting, 2013. Numerical simulations of complex fluid-fluid interface dynamics. The European Physical Journal Special Topics 222:177-198.

23. Helfrich, W., 1973. Elastic properties of lipid bilayers: theory and possible experiments. Z Naturforsch C 28:693-703. https://www.ncbi.nlm.nih.gov/pubmed/4273690.

24. Kaoui, B., G. H. Ristow, I. Cantat, C. Misbah, and W. Zimmermann, 2008. Lateral migration of a two-dimensional vesicle in unbounded Poiseuille flow. Phys Rev E Stat Nonlin Soft Matter Phys 77:021903. https://www.ncbi.nlm.nih. gov/pubmed/18352047.

25. Peskin, C. S., 2002. The immersed boundary method. Acta Numerica 11:479 - 517.

26. Betz, T., M. Lenz, J.-F. Joanny, and C. Sykes, 2009. ATP-dependent mechanics of red blood cells. Proceedings of the National Academy of Sciences 106:15320-15325. https://www.pnas.org/content/106/36/15320. 
Gou et al.

27. Pak, O. S., Y.-N. Young, G. R. Marple, S. Veerapaneni, and H. A. Stone, 2015. Gating of a mechanosensitive channel due to cellular flows. Proceedings of the National Academy of Sciences 112:9822-9827. https://www.pnas.org/ content/112/32/9822.

28. Lumpkin, E. A., and M. J. Caterina, 2007. Mechanisms of sensory transduction in the skin. Nature 445:858-65. https://www.ncbi.nlm.nih.gov/pubmed/17314972.

29. Gov, N. S., and S. A. Safran, 2005. Red blood cell membrane fluctuations and shape controlled by ATP-induced cytoskeletal defects. Biophys J 88:1859-74. https://www.ncbi.nlm.nih.gov/pubmed/15613626.

30. Discher, D. E., and P. Carl, 2001. New insights into red cell network structure, elasticity, and spectrin unfolding - a current review. Cellular and Molecular Biology Letters 6:593-606.

31. Lee, J. C., and D. E. Discher, 2001. Deformation-enhanced fluctuations in the red cell skeleton with theoretical relations to elasticity, connectivity, and spectrin unfolding. Biophys J 81:3178-92. https://www.ncbi.nlm.nih.gov/pubmed/ 11720984.

32. Law, R., P. Carl, S. Harper, P. Dalhaimer, D. W. Speicher, and D. E. Discher, 2003. Cooperativity in forced unfolding of tandem spectrin repeats. Biophys J 84:533-44. https://www.ncbi.nlm.nih.gov/pubmed/12524305.

33. Takeishi, N., M. E. Rosti, Y. Imai, S. Wada, and L. Brandt, 2019. Haemorheology in dilute, semi-dilute and dense suspensions of red blood cells. Journal of Fluid Mechanics 872:818-848.

34. Krüger, T., H. Kusumaatmaja, A. Kuzmin, O. Shardt, G. Silva, and E. M. Viggen, 2017. The Lattice Boltzmann Method. Graduate Texts in Physics. Springer International Publishing, 1 edition.

35. Kaoui, B., and J. Harting, 2015. Two-dimensional lattice Boltzmann simulations of vesicles with viscosity contrast. Rheologica Acta 55:465-475.

36. Zhang, H. D., and C. Misbah, 2019. Lattice Boltzmann simulation of advection-diffusion of chemicals and applications to blood flow. Computers \& Fluids 187:46-59.

37. Suresh, S., 2006. Mechanical response of human red blood cells in health and disease: Some structure-property-function relationships. Journal of materials research 21:1871-1877.

38. John, K., and A. I. Barakat, 2001. Modulation of ATP/ADP concentration at the endothelial surface by shear stress: effect of flow-induced ATP release. Ann Biomed Eng 29:740-51. https://www.ncbi.nlm.nih.gov/pubmed/11599582.

39. Gorman, M. W., E. O. Feigl, and C. W. Buffington, 2007. Human plasma ATP concentration. Clin Chem 53:318-25. https://www.ncbi.nlm.nih.gov/pubmed/17185366.

40. Popel, A. S., and P. C. Johnson, 2005. Microcirculation and Hemorheology. Annu Rev Fluid Mech 37:43-69. https: //www.ncbi.nlm.nih.gov/pubmed/21151769.

41. Xu, S., X. Li, K. B. LaPenna, S. D. Yokota, S. Huke, and P. He, 2017. New insights into shear stress-induced endothelial signalling and barrier function: cell-free fluid versus blood flow. Cardiovasc Res 113:508-518. https://www .ncbi.nlm. nih.gov/pubmed/28158679.

42. Yin, X., T. Thomas, and J. Zhang, 2013. Multiple red blood cell flows through microvascular bifurcations: cell free layer, cell trajectory, and hematocrit separation. Microvasc Res 89:47-56. https://www.ncbi.nlm.nih.gov/pubmed/ 23727384.

43. Farutin, A., Z. Y. Shen, G. Prado, V. Audemar, H. Ez-Zahraouy, A. Benyoussef, B. Polack, J. Harting, P. M. Vlahovska, T. Podgorski, G. Coupier, and C. Misbah, 2018. Optimal cell transport in straight channels and networks. Physical Review Fluids 3. <GotoISI > ://WOS : 000447097300002.

44. Sarelius, I. H., and B. R. Duling, 1982. Direct measurement of microvessel hematocrit, red cell flux, velocity, and transit time. Am J Physiol 243:H1018-26. https://www.ncbi.nlm.nih.gov/pubmed/7149038.

45. Vlahovska, P. M., T. Podgorski, and C. Misbah, 2009. Vesicles and red blood cells in flow: From individual dynamics to rheology. Comptes Rendus Physique 10:775-789. 
46. Farutin, A., and C. Misbah, 2013. Analytical and numerical study of three main migration laws for vesicles under flow. Phys Rev Lett 110:108104. https://www.ncbi.nlm.nih.gov/pubmed/23521303.

47. Kaoui, B., G. Biros, and C. Misbah, 2009. Why do red blood cells have asymmetric shapes even in a symmetric flow? Phys Rev Lett 103:188101. https://www.ncbi.nlm.nih.gov/pubmed/19905834.

48. Guckenberger, A., A. Kihm, T. John, C. Wagner, and S. Gekle, 2018. Numerical-experimental observation of shape bistability of red blood cells flowing in a microchannel. Soft Matter https://www.ncbi.nlm.nih.gov/pubmed/ 29473072.

49. Farutin, A., and C. Misbah, 2014. Symmetry breaking and cross-streamline migration of three-dimensional vesicles in an axial Poiseuille flow. Phys Rev E Stat Nonlin Soft Matter Phys 89:042709. https://www.ncbi.nlm.nih.gov/ pubmed/24827280.

50. Kaoui, B., N. Tahiri, T. Biben, H. Ez-Zahraouy, A. Benyoussef, G. Biros, and C. Misbah, 2011. Complexity of vesicle microcirculation. Phys Rev E Stat Nonlin Soft Matter Phys 84:041906. https://www.ncbi.nlm.nih.gov/pubmed/ 22181174.

51. Tomaiuolo, G., L. Lanotte, G. Ghigliotti, C. Misbah, and S. Guido, 2012. Red blood cell clustering in Poiseuille microcapillary flow. Physics of Fluids 24:051903. <GotoISI > //WOS: 000304826100003.

52. Aouane, O., M. Thiebaud, A. Benyoussef, C. Wagner, and C. Misbah, 2014. Vesicle dynamics in a confined Poiseuille flow: from steady state to chaos. Phys Rev E Stat Nonlin Soft Matter Phys 90:033011. https://www.ncbi.nlm.nih. gov/pubmed/25314533.

53. Hirst, G. D., and T. O. Neild, 1978. An analysis of excitatory junctional potentials recorded from arterioles. J Physiol 280:87-104. https://www.ncbi.nlm.nih.gov/pubmed/690942.

54. Händel, C., B. U. S. Schmidt, J. Schiller, U. Dietrich, T. Möhn, T. R. Kießling, S. Pawlizak, A. W. Fritsch, L.-C. Horn, S. Briest, M. Höckel, M. Zink, and J. A. Käs, 2015. Cell membrane softening in human breast and cervical cancer cells. New Journal of Physics 17:083008.

55. Lei, H., and G. E. Karniadakis, 2013. Probing vasoocclusion phenomena in sickle cell anemia via mesoscopic simulations. Proc Natl Acad Sci U S A 110:11326-30. https://www.ncbi.nlm.nih.gov/pubmed/23798393.

56. Ware, R. E., M. de Montalembert, L. Tshilolo, and M. R. Abboud, 2017. Sickle cell disease. The Lancet 390:311-323.

57. Ellis, C. G., S. Milkovich, and D. Goldman, 2012. What is the efficiency of ATP signaling from erythrocytes to regulate distribution of $\mathrm{O}(2)$ supply within the microvasculature? Microcirculation 19:440-50. https://www.ncbi.nlm.nih. gov/pubmed/22587367.

58. Plank, M. J., D. J. Wall, and T. David, 2006. Atherosclerosis and calcium signalling in endothelial cells. Prog Biophys Mol Biol 91:287-313. https://www.ncbi.nlm.nih.gov/pubmed/16171849.

59. Lohman, A. W., M. Billaud, and B. E. Isakson, 2012. Mechanisms of ATP release and signalling in the blood vessel wall. Cardiovasc Res 95:269-80. https://www.ncbi.nlm.nih.gov/pubmed/22678409.

60. Traub, O., and B. C. Berk, 1998. Laminar shear stress: mechanisms by which endothelial cells transduce an atheroprotective force. Arterioscler Thromb Vasc Biol 18:677-685.

61. Atochin, D. N., and P. L. Huang, 2010. Endothelial nitric oxide synthase transgenic models of endothelial dysfunction. Pflugers Arch 460:965-74. https://www.ncbi.nlm.nih.gov/pubmed/20697735.

62. Tsvirkun, D., A. Grichine, A. Duperray, C. Misbah, and L. Bureau, 2017. Microvasculature on a chip: study of the Endothelial Surface Layer and the flow structure of Red Blood Cells. Sci Rep 7:45036. https://www.ncbi.nlm.nih. gov/pubmed/28338083. 